The Texas Medical Center Library

DigitalCommons@TMC

The University of Texas MD Anderson Cancer Center UTHealth Graduate School of

Biomedical Sciences Dissertations and Theses

(Open Access)
The University of Texas MD Anderson Cancer

Center UTHealth Graduate School of

Biomedical Sciences

\title{
EVALUATION OF THE QUANTITATIVE ACCURACY OF A COMMERCIALLY-AVAILABLE POSITRON EMISSION MAMMOGRAPHY SCANNER
}

Adam Springer

Follow this and additional works at: https://digitalcommons.library.tmc.edu/utgsbs_dissertations

Part of the Diagnosis Commons, Equipment and Supplies Commons, and the Other Medical Sciences Commons

\section{Recommended Citation}

Springer, Adam, "EVALUATION OF THE QUANTITATIVE ACCURACY OF A COMMERCIALLY-AVAILABLE POSITRON EMISSION MAMMOGRAPHY SCANNER" (2010). The University of Texas MD Anderson Cancer Center UTHealth Graduate School of Biomedical Sciences Dissertations and Theses (Open Access). 64.

https://digitalcommons.library.tmc.edu/utgsbs_dissertations/64

This Thesis (MS) is brought to you for free and open access by the The University of Texas MD Anderson Cancer Center UTHealth Graduate School of Biomedical Sciences at DigitalCommons@TMC. It has been accepted for inclusion in The University of Texas MD Anderson Cancer Center UTHealth Graduate School of Biomedical Sciences Dissertations and Theses (Open Access) by an authorized administrator of DigitalCommons@TMC. For more information, please contact digitalcommons@library.tmc.edu.

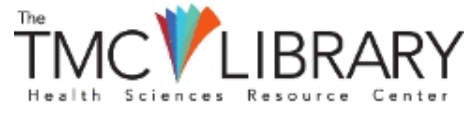




\title{
EVALUATION OF THE QUANTITATIVE ACCURACY OF A COMMERCIALLY- AVAILABLE POSITRON EMISSION MAMMOGRAPHY SCANNER
}

\author{
By
}

Adam C. Springer, B.S.

\section{APPROVED:}

Osama Mawlawi, Ph.D.

Supervisory Professor

Tinsu Pan, Ph.D.

Valen E. Johnson, Ph.D.

Yiping Shao, Ph.D.

Eric Rohren, M.D.

Richard E. Wendt III, Ph.D.

APPROVED:

George Stancel, Ph.D.

Dean, The University of Texas

Graduate School of Biomedical Sciences at Houston 


\title{
EVALUATION OF THE QUANTITATIVE ACCURACY OF A COMMERCIALLY- AVAILABLE POSITRON EMISSION MAMMOGRAPHY SCANNER
}

\author{
A Thesis \\ Presented to the Faculty of \\ The University of Texas \\ Health Science Center at Houston \\ and \\ The University of Texas \\ M.D. Anderson Cancer Center \\ Graduate School of Biomedical Sciences \\ in Partial Fulfillment \\ of the Requirements \\ for the Degree of \\ MASTER OF SCIENCE \\ by \\ Adam C. Springer, B.S. \\ Houston, Texas
}

August, 2010 


\section{ACKNOWLEDGEMENTS}

This thesis would not have been possible without Dr. Osama Mawlawi. His support and encouragement were invaluable and I will remain forever grateful for this opportunity. I would also like to express my appreciation to my committee, whose feedback served to strengthen my research.

This thesis would also not be possible without the PEM Flex system on loan to M.D. Anderson Cancer Center from Naviscan, Inc. I would specifically like to thank Chris Matthews, Weidong Luo and Eileen Lu for their input and assistance.

I appreciate all the help of the PET technologists who facilitated the countless scans I performed: Richelle Millican, Shree Taylor, and Amanda Williams.

I would like to thank my family for all their love through the years. I owe my deepest gratitude to Rebecca and Lizzy whose love and support were instrumental through this process. 


\title{
EVALUATION OF THE QUANTITATIVE ACCURACY OF A COMMERCIALLY-AVAILABLE POSITRON EMISSION MAMMOGRAPHY SCANNER
}

\author{
Publication No.: \\ Adam C. Springer, B.S. \\ Supervisory Professor: Osama Mawlawi, Ph.D.
}

Objective: The PEM Flex Solo II (Naviscan, Inc., San Diego, CA) is currently the only commercially-available positron emission mammography (PEM) scanner. This scanner does not apply corrections for count rate effects, attenuation or scatter during image reconstruction, potentially affecting the quantitative accuracy of images. This work measures the overall quantitative accuracy of the PEM Flex system, and determines the contributions of error due to count rate effects, attenuation and scatter.

Materials and Methods: Gelatin phantoms were designed to simulate breasts of different sizes $(4-12 \mathrm{~cm}$ thick) with varying uniform background activity concentration $(0.007-0.5 \mu \mathrm{Ci} / \mathrm{cc})$, cysts and lesions $(2: 1,5: 1,10: 1$ lesion-to-background ratios). The overall error was calculated from ROI measurements in the phantoms with a clinically relevant background activity concentration $(0.065 \mu \mathrm{Ci} / \mathrm{cc})$. The error due to count rate effects was determined by comparing the overall error at multiple background activity concentrations to the error at $0.007 \mu \mathrm{Ci} / \mathrm{cc}$. A point source and cold gelatin phantoms were used to assess the errors due to attenuation and scatter. The maximum pixel values in gelatin and in air were compared to determine the effect of attenuation. Scatter was evaluated by comparing the sum of all pixel values in gelatin and in air.

Results: The overall error in the background was found to be negative in phantoms of all thicknesses, with the exception of the 4 -cm thick phantoms $(0 \% \pm 7 \%)$, 
and it increased with thickness (-34\% $\pm 6 \%$ for the $12-\mathrm{cm}$ phantoms). All lesions exhibited large negative error $(-22 \%$ for the $2: 1$ lesions in the $4-\mathrm{cm}$ phantom) which increased with thickness and with lesion-to-background ratio (-85\% for the 10:1 lesions in the $12-\mathrm{cm}$ phantoms). The error due to count rate in phantoms with $0.065 \mu \mathrm{Ci} / \mathrm{cc}$ background was negative $(-23 \% \pm 6 \%$ for 4 -cm thickness) and decreased with thickness $(-7 \% \pm 7 \%$ for 12 $\mathrm{cm})$. Attenuation was a substantial source of negative error and increased with thickness $(-51 \% \pm 10 \%$ to $-77 \% \pm 4 \%$ in 4 to $12 \mathrm{~cm}$ phantoms, respectively). Scatter contributed a relatively constant amount of positive error $(+23 \% \pm 11 \%)$ for all thicknesses.

Conclusion: Applying corrections for count rate, attenuation and scatter will be essential for the PEM Flex Solo II to be able to produce quantitatively accurate images. 


\section{TABLE OF CONTENTS}

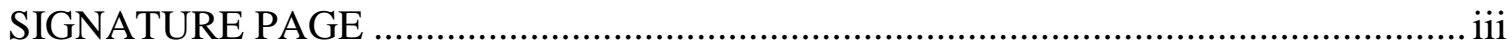

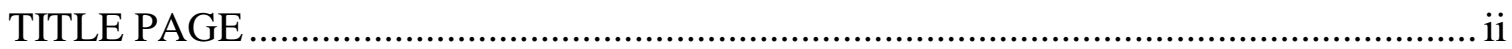

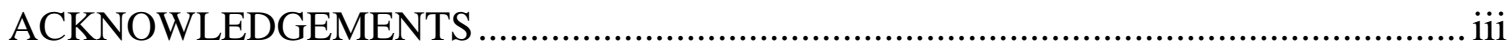

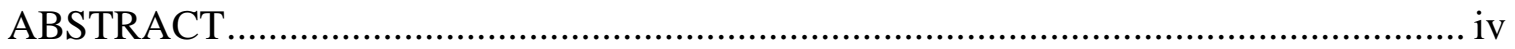

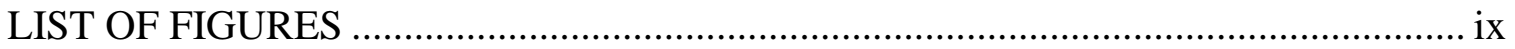

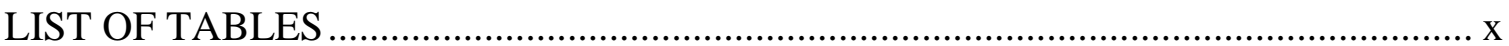

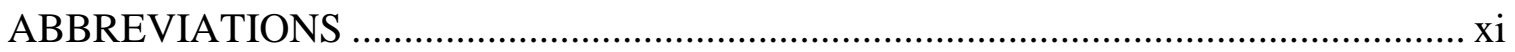

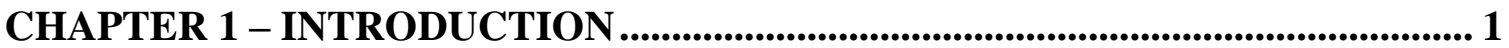

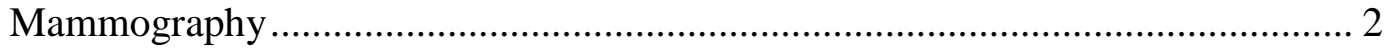

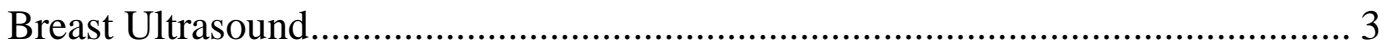

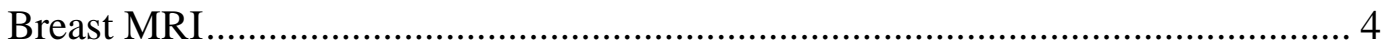

Functional Breast Imaging ...................................................................... 4

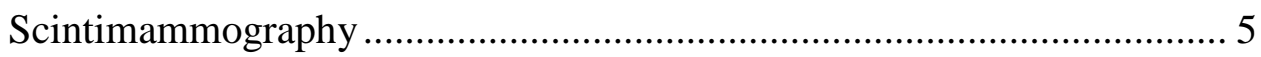

Molecular Breast Imaging/Breast-Specific Gamma Imaging ..................... 5

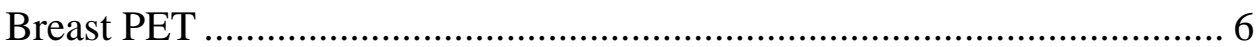

Positron Emission Mammography........................................................... 6

Hypothesis and Specific Aims .......................................................................... 8

CHAPTER 2 - MATERIALS AND METHODS............................................................ 9

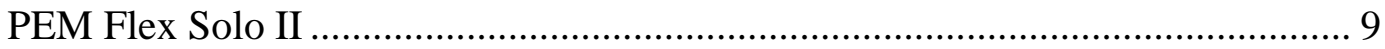




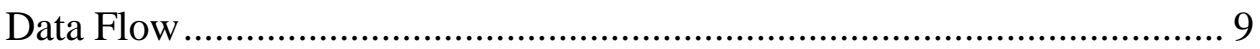

System Characterization ……………………………………………..... 11

Specific Aim 1: Total Quantitative Error............................................................. 12

Gelatin Breast Phantoms .................................................................... 12

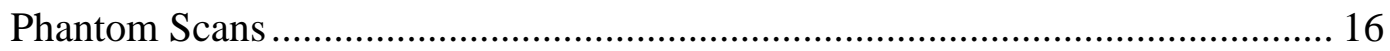

Calculation of Total Quantitative Error ................................................... 17

Specific aim 2: Error due to Count Rate ............................................................. 18

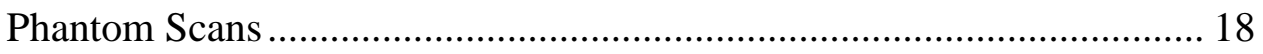

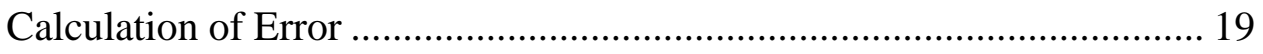

Specific Aim 3: Error due to Attenuation .......................................................... 20

Point Source Transmission Scans ......................................................... 21

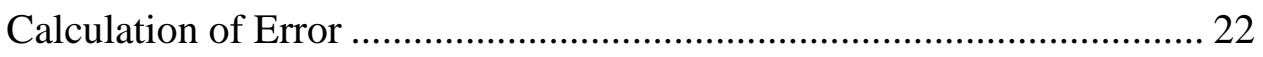

Specific Aim 4: Error due to Scatter.................................................................. 23

Point Source Transmission Scans ............................................................. 23

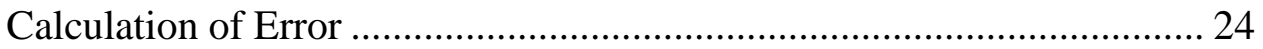

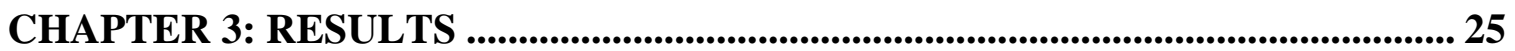

Specific Aim 1: Total Quantitative Error............................................................... 25

Specific Aim 2: Error due to Count Rate ............................................................. 27

Specific Aim 3: Error due to Attenuation ............................................................... 29 
Specific Aim 4:Error due to Scatter.

CHAPTER 4 - DISCUSSION AND CONCLUSIONS............................................. 32

Specific Aim 1: Total Quantitative Error................................................................. 32

Specific Aim 2: Error due to Count Rate............................................................. 34

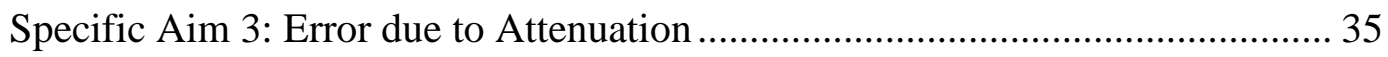

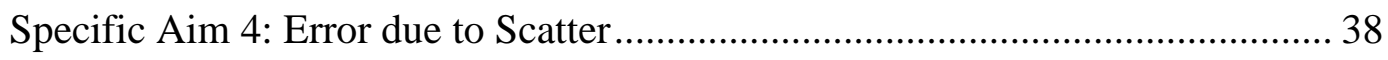

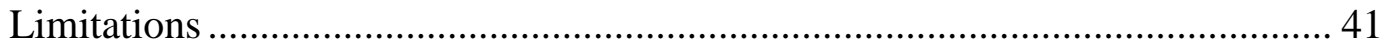

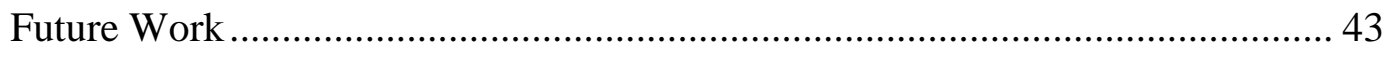

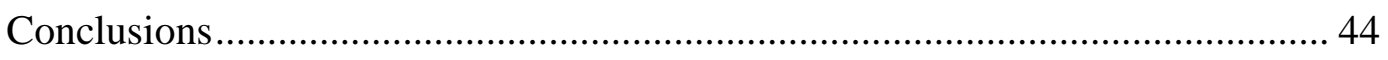

REFERENCES......................................................................................................................... 46

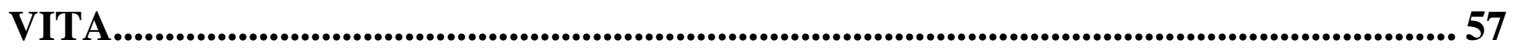




\section{LIST OF FIGURES}

Figure 1.1: The Naviscan PEM Flex Solo II positron emission mammography scanner... 8

Figure 2.1: Gelatin breast phantoms ................................................................ 14

Figure 2.2: Reference and transmission scans of a point source for measuring the error due to attenuation

Figure 3.1: The total quantitative error in images of 4, 6, 8, 10 and 12-cm thick gelatin breast phantoms with simulated cysts and lesions of different lesion-to-background ratios $(2: 1,5: 1,10: 1)$ 26

Figure 3.2: Images of lesions and cysts in gelatin breast phantoms of each thickness with $0.065 \mu \mathrm{Ci} / \mathrm{cc}$ background AC. Images are displayed with the same window width and level $(14,000 / 7,000 \mathrm{~Bq} / \mathrm{ml})$ 26 Figure 3.3: All 12 PEM images (1-cm thick) of a 12-cm thick phantom with 5:1 and 10:1 lesions in a uniform background $\mathrm{AC}$ of $0.065 \mu \mathrm{Ci} / \mathrm{cc}$ 27

Figure 3.4: Error due to count rate in uniform background of phantoms with varying

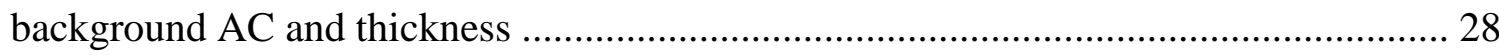

Figure 3.5: Error due to count rate in lesions and background of phantoms with 0.065 $\mu \mathrm{Ci} / \mathrm{cc}$ background $\mathrm{AC}$ 29

Figure 3.6: The measured error due to attenuation with the percent signal loss expected due to attenuation of $511 \mathrm{keV}$ photons in the given thicknesses (D) of water ............... 30

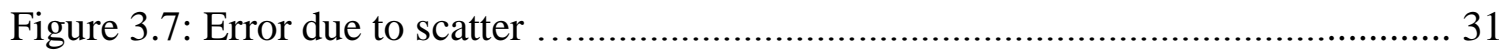

Figure 4.1: Path length and attenuation of oblique LORs ........................................ 37

Figure 4.2: The signal loss due to attenuation along the most oblique LORs ............... 37

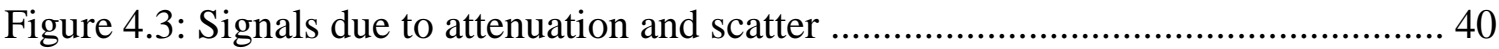




\section{LIST OF TABLES}

Table 2.1: Typical Clinical PEM Protocol ...................................................................... 11

Table 2.2: Linear Attenuation Coefficients of Water and Breast Tissue ........................ 14

Table 2.3: Estimated Background Activity Concentration in Normal Breast Tissue ...... 15 


\section{ABBREVIATIONS}

18F-FDG - 2- $\left[{ }^{18}\right.$ F]-fluoro-2-deoxy-D-glucose

$\mathrm{AC}-$ Activity Concentration

ACS - American Cancer Society

CBE - Comprehensive Breast Exam

CT - Computed Tomography

DOI - Depth of Interaction

FOV - Field of View

$\mathrm{keV}$ - Kiloelectronvolt

LAT - Limited-angle Tomography

LBR - Lesion-to-Background Ratio

LOR - Line of Response

NEMA - National Electrical Manufacturer's Association

PEM - Positron Emission Mammography

PERCIST - Positron Emission Response Criteria in Solid Tumors

PET - Positron Emission Tomography

PPV - Positive Predictive Value

PVE - Partial Volume Effect

ROI - Region of Interest

$\mathrm{SF}$ - Scatter Fraction 


\section{CHAPTER 1 - INTRODUCTION}

Breast cancer is by far the most commonly diagnosed non-skin cancer in women in the United States, and it is the second most fatal cancer in that population. It was estimated that 192,370 new cases of breast cancer would be diagnosed in women in the United States in 2009, and that 40,170 women would die from the disease(1)(1) (1). It has been shown that the mortality due to breast cancer varies with stage at the time of diagnosis (2). Between 1999 and 2006, the 5-year relative survival of patients diagnosed with localized disease was $98 \%$. This decreased to $84 \%$ in patients whose disease had spread to regional lymph nodes, and to $23 \%$ in patients with metastatic disease. Early detection of breast cancer is clearly vital to improving survival by allowing intervention during earlier stages of disease. Additionally, early detection and accurate diagnosis may afford patients more treatment options, such as breast conserving surgery (lumpectomy vs. mastectomy) and less aggressive adjuvant chemotherapy (3).

Mammography is now the primary imaging modality for breast cancer screening and its widespread use is responsible for reducing the mortality of breast cancer $(4,5)$. As with any test, mammography has certain limitations, particularly lower sensitivity in women with dense breasts and low positive predictive value (PPV) overall (6). Other disadvantages of mammography include exposure to ionizing radiation and patient discomfort. Using adjunct imaging modalities (e.g. ultrasound, MRI and functional imaging) in combination with mammography improves the sensitivity of breast cancer screening compared with mammography alone $(7,8)$. The benefits and limitations of 
mammography and some common adjunctive modalities for imaging breast cancer are discussed below.

\section{Mammography}

Mammography is the application of projection radiography to breast imaging. The radiographic appearance of normal breast tissue and cancers in the breast was first studied ex vivo in the 1910s (9) and clinical investigations with mammography in the United States were reported in 1930 (10). The diagnostic value of mammography was not recognized until the early 1950s but still was not used by most radiologists into the 1960s because of technical difficulties and limitations, in addition to its lack of reliability and reproducibility (11). Owing to advances in mammography since then, multiple large, randomized clinical trials have shown that mammography screening substantially reduces the mortality of breast cancer through early detection and intervention $(4,5)$. Such results inspired the American Cancer Society (ACS) to recommend that women who are over the age of 40 years and have an average risk of developing breast cancer receive annual screening mammograms (3).

Mammography has become the primary imaging modality for breast cancer screening because of its high sensitivity, and it plays an important role in diagnosis. Although the sensitivity is high overall, it varies with breast compositions and it is lower in dense breasts $(6,12)$. Digital mammography has slightly better sensitivity than filmscreen mammography in certain subgroups, particularly women with heterogeneously dense or very dense breasts $(13,14)$. Regardless of detector type, one weakness of mammography is the 2D nature of projection imaging, because overlying tissue can 
obscure or mask lesions. Also, the specificity and PPV are low $(4,6)$, leading to many unnecessary diagnostic procedures such as additional mammographic views, fine needle aspirations or ultrasound-guided core biopsies. Fortunately, there are other diagnostic modalities that do not share the same limitations as mammography.

\section{Breast Ultrasound}

With respect to breast imaging, one advantage of ultrasound (US) is that lesions are not obscured by overlying tissue. Also, US does not expose the patient to ionizing radiation. US is commonly used to characterize suspicious lesions found with mammography, or when palpable masses are not visible in mammograms (15). US can easily differentiate between fluid-filled cysts and solid nodules (16) and using strict criteria such as the BI-RADS lexicon has allowed some solid nodules to be classified as benign or malignant with US $(17,18)$. If lesions cannot be ruled out as benign, US is also useful for guiding needle core biopsies without unnecessarily exposing patients to ionizing radiation. In addition to its roles in diagnosis and follow-up, US could be a useful adjunct to mammography for breast cancer screening, particularly in dense breasts $(6,7)$. While US cannot easily detect microcalcifications indicative of DCIS (19), early stage, node-negative cancers have been discovered with US (20-24). Thus, the use of adjunct US for breast cancer screening increases the sensitivity of detecting breast cancer to earlier stage cancers than mammography alone. Whereas the sensitivity is greater, the reported specificity and positive predictive value (PPV) are often lower because many of the additional findings are benign. The low specificity and high false positive rate are two reasons US screening has not been widely adopted as a standard procedure. 


\section{$\underline{\text { Breast MRI }}$}

Magnetic resonance imaging (MRI) can acquire volumetric images, eliminating the problem of overlying tissue. Ionizing radiation is also not of concern in MRI. Like US, MRI is commonly used to follow up on suspicious mammographic findings. Breast MRI is useful for determining whether diagnosed breast cancers have spread beyond what is indicated with mammography or US, e.g. multifocal or multicentric disease, nodal involvement or chest wall invasion (15). Breast MRI can also detect cancers which are mammographically and clinically occult (25) and it may used to guide biopsies of breast lesions (26). Due to its high sensitivity, breast MRI is suggested by the ACR as an adjunct to mammography for screening of certain high-risk women (3). However, many benign lesions and even normal breast tissue can be mistaken for cancer, leading to very low specificity of this modality $(25,27)$.

\section{Functional Breast Imaging}

Functional imaging is yet another tool available for imaging breast cancer with the advantage of supplementing the anatomical imaging modalities with information about the disease state. Overlying tissue is less likely to obscure lesions, though it might reduce the signal. Conventional nuclear medicine modalities vary in their sensitivities to breast cancer due to the resolution of conventional systems, but they have higher specificity than anatomical modalities (8). High-resolution imaging devices are under investigation and have improved detection sensitivity in small tumors. 


\section{Scintimammography}

Scintimammography is a functional imaging technique that commonly uses

${ }^{99 \mathrm{~m}}$ Tc-methoxyisobutylisonitrile $\left({ }^{99 \mathrm{~m}} \mathrm{Tc}\right.$-sestamibi) to image breast cancer $(8) .{ }^{99 \mathrm{~m}} \mathrm{Tc}-$ sestamibi concentrates in breast cancers (28) due to an increase in blood flow, number of mitochondria, membrane hyperpolarization in the tumor or expression of the multidrug resistance gene (29-32). Patients are imaged prone with the breast pendant and uncompressed (8). Scintimammography has good overall sensitivity and specificity to breast cancer $(33,34)$. The resolution of conventional gamma cameras limits the sensitivity of scintimammography to tumors smaller than $5 \mathrm{~mm}$ and it is more useful for imaging palpable primary breast cancers than non-palpable ones (28).

\section{Molecular Breast Imaging/Breast-Specific Gamma Imaging}

Molecular Breast Imaging (MBI) and Breast-Specific Gamma Imaging (BSGI) acquire images of ${ }^{99 \mathrm{~m}} \mathrm{Tc}$-sestamibi with small gamma cameras dedicated to breast imaging (35). These systems use mild compression to immobilize the breast and decrease the amount of attenuating tissue between lesions and the detector, can be positioned much closer to the breast and have much better spatial resolution, thus offering better sensitivity to small tumors than conventional gamma cameras. Studies at the Mayo clinic have shown that MBI has similar sensitivity to breast MRI (35). It also detected 2- to 3-times more cancers in dense breasts than mammography, and with slightly better specificity. 


\section{Breast PET}

Another functional imaging modality is positron emission tomography (PET), which has the advantage of quantifying activity concentration. PET is most commonly performed with the radiopharmaceutical 2-[ $\left.{ }^{18} \mathrm{~F}\right]$-fluoro-2-deoxy-D-glucose (FDG), a glucose analog which is trapped in metabolically active cells (36). Cancer cells typically have a higher metabolic rate than normal tissue, resulting in greater FDG uptake in malignancies. The quantitative nature of PET allows accurate diagnosis or staging of advanced breast cancers $(37,38)$ as well as monitoring of treatment response $(39,40)$. The resolution of conventional scanners limits the sensitivity of PET to tumors greater than 5-10 mm.

\section{Positron Emission Mammography}

To overcome the limitations of whole body PET scanners for imaging breast cancer, Thompson, Murth, Weinberg and Mako (41) designed a dedicated scanner with a more optimal geometry for high detector efficiency and high resolution. The original positron emission mammography (PEM) scanner used dual-head opposing planar coincidence detectors between which a breast could be immobilized with mild compression. Several PEM systems with variations of Thompson's configuration have been investigated since at least the early 2000s (42-47).

Using dedicated detectors very close to or in contact with the breast being imaged is extremely advantageous in two ways. First, these systems have greater geometric efficiency due to the large solid angle subtended by the detectors. Thus, the detection efficiency is on the order of two orders of magnitude greater than whole body 3D PET 
scanners (48). The compression used by most systems reduces attenuation, which also increases the detection efficiency. Second, the small distance between the detectors reduces blurring due to noncolinearity to less than $0.25 \mathrm{~mm}$-much smaller than a pixel-for even a $10 \mathrm{~cm}$ thick breast (49). Employing small crystals in this detector configuration further improves the resolution over whole-body PET scanners $(<2.5 \mathrm{~mm}$ vs. > 4 mm FWHM) $(7,41,43,45,50)$.

These impressive performance characteristics allow PEM to detect breast cancer earlier than conventional PET, including in situ cancers $(45,50,51)$. In addition to high sensitivity, PEM has high specificity $(50,52)$. Other benefits of PEM include correlation with $\mathrm{x}$-ray mammograms by incorporating both imaging devices into one system, as well as image-guided biopsies (53). While PEM is very useful for detecting breast cancer, accurate diagnosis and staging with PEM will require systems to accurately quantify radiotracer uptake.

There is currently only one commercially-available PEM scanner, the PEM Flex Solo II (Naviscan, Inc., San Diego, CA). This system is described in Chapter 2, but briefly, it uses opposing planar coincidence detectors between which a patient's breast is immobilized during scans with mild compression $(48,54)$. Corrections such as for dead time, randoms, attenuation and scatter are not currently applied on the PEM Flex. While this scanner is very good at detecting disease (50), accurate diagnosis and staging based on radiotracer uptake will require the system to be quantitatively accurate.

The purpose of this thesis is to evaluate the quantitative accuracy of the PEM Flex Solo II, and to determine the contributions of error from count rate effects, attenuation and scatter. As a benchmark for accuracy, the Positron Emission Response Criteria In 
Solid Tumors (PERCIST) suggests that treatment response of a tumor is indicated by PET if SUV decreases by $30 \%$ (55).

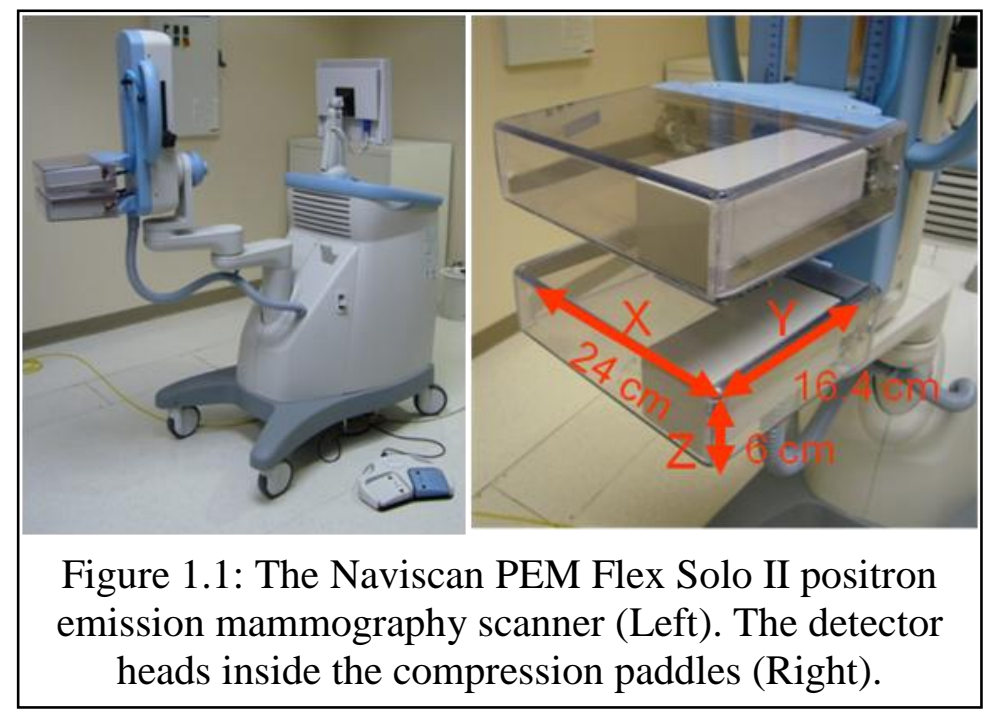

\section{Hypothesis and Specific Aims}

Hypothesis: The total quantitative error of images acquired with the PEM Flex Solo II is greater than $30 \%$, due in part to count rate effects, attenuation and scatter.

This hypothesis was tested using the following specific aims:

1) Measure the total quantitative error in breast phantoms of multiple sizes, with uniform background and embedded cysts and lesions of different lesion-tobackground ratios

2) Determine the error due to count rate effects

3) Determine the error due to attenuation

4) Determine the error due to scatter 


\section{CHAPTER 2 - MATERIALS AND METHODS}

\section{$\underline{\text { PEM Flex Solo II }}$}

\section{System Configuration}

The PEM Flex Solo II scanner utilizes a scanning, dual-headed coincidence detector to produce limited-angle tomographic (LAT) images. The detectors are mounted on an articulating arm which allows images to be acquired in any orientation, e.g. craniocaudal and mediolateral. The lower (support) paddle is fixed to the arm while the upper (compression) paddle is adjustable to provide mild compression (15 lbs of force) and can be moved up to $20 \mathrm{~cm}$ from the support paddle.

The detectors are housed inside $16.8 \times 6.2 \times 5.5 \mathrm{~cm}^{3}$ enclosures which scan synchronously across the FOV in the x-direction during acquisitions. The enclosure is light-tight and EMI-tight. Additionally, the enclosure is $95 \%$ tungsten on 5 sides to shield the detectors from radiation outside the FOV. The entrance window is 1-m thick aluminum to maximize transmission of annihilation photons from within the FOV. Each detector head houses a $2 \times 6$ matrix of detector modules, each of which comprises a crystal array, a reflective light guide and a position-sensitive photomultiplier tube (PSPMT). Individual crystals $\left(2 \times 2 \times 12 \mathrm{~cm}^{3}\right)$ of LYSO are packed in $13 \times 13$ arrays with a crystal pitch of $2.1 \mathrm{~mm}$.

\section{Data Flow}

During acquisition, PMT signals are tested for coincidence with a coincidence timing window of $6 \mathrm{~ns}$. List data for coincident events include a time stamp and 32 ADC 
values. A delayed coincidence window of about $100 \mathrm{~ns}$ is used to estimate random events, which are written to their own list file but not used to correct for randoms.

Table 2.1: Typical Clinical PEM Protocol

\begin{tabular}{|c|c|}
\hline \multicolumn{2}{|c|}{ Acquisition } \\
\hline Views & $\begin{array}{l}\text { Mediolateral and craniocaudal of } \\
\text { affected breast(s) }\end{array}$ \\
\hline Field-of-view (x-y plane) & $24 \times 16.8 \mathrm{~cm}^{2}($ maximum $)$ \\
\hline FOV (z-direction) & Patient dependent (up to $19 \mathrm{~cm}$ ) \\
\hline Compression force & $\leq 15 \mathrm{lbs}$ \\
\hline Scan duration & Variable (typically $10 \mathrm{~min}$ ) \\
\hline \multicolumn{2}{|c|}{ Reconstruction } \\
\hline Coincidence timing window & $6 \mathrm{~ns}$ \\
\hline Energy window & $350-750 \mathrm{keV}$ \\
\hline Acceptance angle & $\begin{array}{l}25 \text { crystals. Angle varies with } \\
\text { paddle separation }\end{array}$ \\
\hline Algorithm & $\begin{array}{l}\text { Iterative 3D Maximum Likelihood } \\
\text { Expectation Maximization }\end{array}$ \\
\hline Number of iterations & 5 \\
\hline Corrections & $\begin{array}{l}\text { Detector normalization, geometric } \\
\text { efficiency. NO corrections for } \\
\text { randoms, dead-time, attenuation, } \\
\text { scatter or intrascan decay }\end{array}$ \\
\hline Reconstruction time & $\begin{array}{l}\text { Depends on number of counts } \\
\text { (typically }<15 \mathrm{~min} \text { ) }\end{array}$ \\
\hline \multicolumn{2}{|r|}{ Images } \\
\hline Image matrix & $136 \times 200$ \\
\hline Pixel size & $1.2 \times 1.2 \mathrm{~mm}^{2}$ \\
\hline Resolution & $2.4 \mathrm{~mm}$ FWHM \\
\hline Number of slices & 12 \\
\hline Slice thickness & $1 / 12^{\text {th }}$ detector separation \\
\hline Units & $\begin{array}{l}\mu \mathrm{Ci} / \mathrm{cc} \text { or PEM Uptake Value } \\
(\mathrm{PUV})\end{array}$ \\
\hline
\end{tabular}

The ADC values are used subsequently to identify the coordinates and energy of each event during rebinning, at which point an energy window is applied. The default lower and upper level discriminators (LLD and ULD) are 350 and $750 \mathrm{keV}$, respectively. Coincident events within the energy window are rebinned into a 4D histogram and written to a decode file which is used for reconstruction. 
Images are reconstructed from data in the decode file using an iterative, 3D maximum-likelihood expectation maximization (MLEM) algorithm. In standard clinical mode 5 iterations are used to reconstruct 24 evenly-spaced image planes. The slice thickness is equal to $1 / 24^{\text {th }}$ of the detector separation. The maximum acceptance angle is determined by crystal separation and detector separation. In the $\mathrm{x}$-direction the crystal separation is physically limited by the width of the detector heads $(5.5 \mathrm{~cm}$, or 26 crystal). There are 78 crystals in the y-direction, but the default constraint on acceptance angle is the same as in the $\mathrm{x}$-direction.

The PEM Flex system currently applies no corrections for count rate effects, attenuation or scatter. Corrections which are performed are detector normalization and decay correction to injection time (only for PUV images). No corrections are applied for intrascan decay, which is $8.7 \%$ over 10 minutes for $18 \mathrm{~F}$.

Rebinning and reconstruction begin during acquisition and partially reconstructed images are displayed. List data are rebinned in approximately one-minute "chunks" from which images are reconstructed independently of other chunks. After each chunk is reconstructed, the new image is merged with all previously reconstructed chunks.

\section{System Characterization}

Measurements characterizing the system performance have been reported by others (45), including the manufacturer (48). With the paddles separated by $9 \mathrm{~cm}$, the resolution was about $2.4 \mathrm{~mm}$ in-plane and more than $9 \mathrm{~mm}$ in the direction perpendicular to the detectors. The total sensitivity was found to be $0.16 \mathrm{cps} / \mathrm{Bq}$ using a point source inair with the paddle separation set to $5 \mathrm{~cm}$. The scatter fraction using a line source in a rat phantom was $13 \%$. Uniformity in images of a 5 -ml intravenous saline bag with 18F-FDG 
was reported to be $6 \%$ by one group (45). Linearity and NECR were also evaluated as well as recovery coefficients for objects as small as $1 \mathrm{~mm}$.

\section{Specific Aim 1: Total Quantitative Error}

\section{Gelatin Breast Phantoms}

To measure the overall quantitative error of the PEM Flex Solo II (described in the Introduction), phantoms were needed which satisfied several criteria. While the SUV in breasts varies with tissue type (56), a uniform background activity concentration (hereafter referred to as background) is more reproducible than a heterogeneous distribution and it reduces measurement uncertainty. Further, the actual background in phantoms should be representative of that observed clinically. Breast cancers exhibit a range of lesion-to-background ratios (LBR) and cysts with no uptake may also be present, thus multiple LBRs and cysts needed to be simulated. The linear attenuation and scatter coefficients of the phantoms needed to be comparable to breast tissue at $511 \mathrm{keV}$. In addition to the radiological properties of the material, the size and shape of an object may influence how much attenuation and scatter contribute to the error. Thus, a range of sizes was needed as well as a shape similar to that of a breast under mild compression between the detector paddles.

An alternative to water which has previously been used to construct PEM phantoms is gelatin $(44,57)$. Gelatin is well-suited for breast phantoms in part because, like water, the background and size are arbitrary. In addition, gelatin can be made in an arbitrary shape, and simulated lesions and cysts can be positioned anywhere inside the 
phantoms without introducing other materials such as hollow plastic spheres. Also, as noted below, the linear attenuation coefficient of the gelatin was within $1.5 \%$ of those of water at diagnostic energies. This difference should be negligible at $511 \mathrm{keV}$, thus gelatin was assumed to be radiologically similar to breast tissue at $511 \mathrm{keV}$ (Table 2.2).

Table 2.2: Linear Attenuation Coefficients of Water and Breast Tissue

\begin{tabular}{l|c|c}
\multicolumn{1}{c|}{ Material } & $\begin{array}{c}\text { Total } \\
\mu\left(\mathrm{cm}^{-1}\right)\end{array}$ & $\begin{array}{c}\text { Compton } \\
\mu\left(\mathrm{cm}^{-1}\right)\end{array}$ \\
\hline Breast Tissue (ICRU-44) & 0.0973 & 0.0971 \\
\hline Water & 0.0959 & 0.0956 \\
\hline Percent Difference & $-1.4 \%$ & $-1.5 \%$ \\
\hline
\end{tabular}

The elemental composition and density $\left(1.02 \mathrm{~g} / \mathrm{cm}^{3}\right)$ of breast tissue comprising 50\% adipose and 50\% glandular tissue reported in ICRU 44 were entered into XCOM (58) to calculate the mass attenuation and incoherent (Compton) scattering coefficients, from which the linear coefficients were calculated. The coefficients for water were also calculated in XCOM using the elemental composition of $\mathrm{H}_{2} \mathrm{O}$.

The phantoms used to measure the overall quantitative error comprised stackable, semicircular slabs of gelatin with uniform background and gelatin cysts and lesions. The background activity concentration was chosen to be $0.065 \mu \mathrm{Ci} / \mathrm{cc}(2590 \mathrm{~Bq} / \mathrm{ml})$, to approximate the background in normal breast tissue during conventional PET scans (Table 2.3). Each phantom contained either two cysts and two 2:1 lesions, or two 5:1 and two 10:1 lesions (Figure 2.1a) fully embedded in the middle layer, for a total of four objects in each phantom. The objects were spaced to minimize the signal each would contribute to measurements in the others and in the uniform background. The slabs were easily reproducible, could be stacked to the desired heights $(4,6,8,10$ and $12 \mathrm{~cm})$, and 
the profile of each phantom resembled the shape of a breast compressed between the detectors (Figure 2.1b).

Table 2.3: Estimated Background Activity Concentration in Normal Breast Tissue

\begin{tabular}{l|c}
\hline Injected Dose (59) & $15 \mathrm{mCi}$ \\
\hline Patient Mass (60) & $74 \mathrm{~kg}$ \\
\hline Uptake Time (55) & $60 \mathrm{~min}$ \\
\hline SUV (61) & 0.49 \\
\hline Activity Background Concentration & 0.067
\end{tabular}

\begin{tabular}{|l|}
\hline A \\
Figure 2.1: Gelatin breast phantoms. A) Simulated lesions \\
and cysts were completely embedded in the middle slab of \\
each phantom. B) Slabs were stacked to 4, 6, 8, 10 and 12 \\
cm and resembled a breast compressed between the \\
detector paddles. C) An assembled phantom positioned on \\
the scanner.
\end{tabular}

To make the phantoms, first gelatin mix (Kroger, Cincinnati, $\mathrm{OH}$ ) was dissolved per the manufacturer's instructions in enough water to make the background lesions and cysts for one phantom. The objects to be embedded - either cysts and 2:1 lesions, or 5:1 and 10:1 lesions-were made first with $100 \mathrm{ml}$ of the gelatin solution for each type of object. Food dye was added to distinguish the objects from each other and activity was added to yield the necessary LBRs. The solution for each type of object was poured into separate $10 \times 1.5 \mathrm{~cm}$ Petri dishes (BD Biosciences, Bedford, MA) and allowed to solidify in a freezer at $-20^{\circ} \mathrm{C}$. The dishes were removed after about 10 minutes, before the gelatin 
froze. The end of a 60-cc syringe was cut off and the edge around the open end was sharpened to be able to cut $3-\mathrm{cm}$ diameter cylinders out of each dish. At $1.5 \mathrm{~cm}$ tall, the lesions and cysts were sufficiently large to avoid partial volume effects, even in the largest phantoms. Two of each type of object were positioned in a $20-\mathrm{cm}$ diameter, disposable plastic plate (Kroger, Cincinnati, $\mathrm{OH}$ ). The plates were clear so the objects could be placed according to a printed template underneath the plate. The arrangement was intended to allow multiple measurements for each type of object in each phantom while minimizing the signal the lesions would contribute to each other and to the background.

18F-FDG was then added to the rest of the gelatin solution and mixed well to yield a uniform background activity concentration (AC). The background gelatin was poured into several disposable plastic plates and allowed to solidify in a freezer. The plates were removed after about 20 minutes, before the gelatin froze. The remainder of the background gelatin was allowed to cool to room temperature before pouring it into the plate with the lesions and cysts, which were kept in a refrigerator in the meantime. This prevented the objects from melting and dissolving into the background. The background gelatin was deep enough to cover the lesions and cysts so they were fully embedded in the slab. As mentioned, each phantom contained for objects-either two cysts and two 2:1 lesions, or two 5:1 and two 10:1 lesions.

Once solid, the slabs of gelatin were cut into two pieces. The bottoms of the plates were flat and the edges were sloped such that the profile of the assembled phantoms resembled a breast compressed between the detector paddles (Figure 2.1b). Prior to scanning the phantoms, each slab was placed in a 1-gallon Ziploc® bag (S.C. Johnson \& 
Son, Inc., Racine, WI) to facilitate handling and to avoid radioactive contamination in case any gelatin broke off.

To confirm that the phantoms were radiologically similar to water, the average CT number was measured in 3 separate $10-\mathrm{cm}$ phantoms. Each phantom was centered on the patient table of a GE Discovery PET/CT scanner (Waukesha, WI) with the slabs aligned transaxially. CT scans $(120 \mathrm{kV}, 300 \mathrm{~mA} 3.75 \mathrm{~mm}$ image thickness, pitch $=1$, standard reconstruction algorithm) were acquired and a large ROI drawn in the middle layer of each. The average pixel value was approximately 15 Hounsfield Units, representative of a $1.5 \%$ greater attenuation coefficient than water for a diagnostic beam. It is expected that this difference is negligible at $511 \mathrm{keV}$ so the gelatin phantoms were assumed to be radiologically similar to breast tissue at that energy.

\section{Phantom Scans}

Three phantoms of each thickness $(4,6,8,10$ and $12 \mathrm{~cm})$ were scanned on the PEM Flex system following a schedule which was intended to be efficient. Phantoms of multiple thicknesses were assembled from each batch of gelatin and scanned serially in order of decreasing thickness. For instance, 10-, 8- and 6-cm thick phantoms were made from a single batch of gelatin. Similarly, $12-\mathrm{cm}$ and $4-\mathrm{cm}$ thick phantoms were made from one batch of gelatin. Thus, a total of 30 scans ( 3 scans $\times 5$ thicknesses $\times 2$ sets of objects) were acquired with 6 batches of gelatin. Due to interscan decay, the background activity of each phantom was within $15 \%$ of the nominal background $(0.065 \mu \mathrm{Ci} / \mathrm{cc})$. 


\section{Calculation of Total Quantitative Error}

Images were reconstructed with the standard clinical protocol (Table 2.1) and evaluated on the scanner GUI in units of $\mu \mathrm{Ci} / \mathrm{cc}$. Region of interest (ROI) measurements were made in the plane at the level of the lesions and cysts. The mean pixel values were measured in $1-\mathrm{cm}^{2}$ ROIs in the uniform region of the background and in the cysts. A large ROI was drawn around each lesion and the maximum pixel value measured. The maximum pixel value was chosen to evaluate the lesion error because the maximum standardized uptake value (SUV) is the most commonly reported metric to assess tumors with PET (55). The error in the background and lesions was calculated as the percent difference between the ROI measurements and the true values (Equation 2.1)

$$
\% \text { Error }=100 \times\left(A C_{R O I}-A C_{\text {True }}\right) / A C_{\text {True }}
$$

where $A C_{R O I}$ is either the mean pixel value in the background or the maximum pixel value in the lesions and $A C_{\text {True }}$ is the respective known activity concentration in each.

The error in the cysts could not be calculated using Equation 2.1 because dividing by the true activity concentration $(0 \mu \mathrm{Ci} / \mathrm{cc})$ would be undefined. Instead, the contrast error in the cysts (Equation 2.2) was calculated from the mean pixel value in a $1-\mathrm{cm}^{2}$ ROI.

$$
\% \text { Contrast } \text { Error }_{\text {Cysts }}=100 \times\left[\left(R O I_{B k g}-R O I_{C y s t}\right) /\right. \text { True Bkg - 1] }
$$

where $R O I_{B k g}$ is the measured background, $R O I_{C y s t s}$ is the mean pixel value in each cyst, and True Bkg is the known activity concentration in the background. 


\section{Specific aim 2: Error due to Count Rate}

To evaluate the count rate behavior of conventional PET scanners, phantoms are commonly made with very high uniform background activity of a short-lived

radioisotope $\left(\right.$ e.g. $\left.{ }^{18} \mathrm{~F}\right)$ and scanned over the course of several half-lives. At very low count rates the net count rate is approximately equal to the true event rate because dead time and randoms are minimal. Therefore the ideal true count rate at all activities is estimated by linear extrapolation from the net count rates for the lowest amounts of activity (62).

This approach was adopted to measure the error in images due to count rate. The error was measured in images of breast phantoms with multiple levels of radioactivity. The error in the lowest activity phantoms was used as a reference to calculate the error contributed by count rate alone at higher background activity concentrations.

\section{Phantom Scans}

The phantoms made to measure the total quantitative error (Specific Aim 1) were also used to measure the error due to count rate. The phantoms which had uniform background $\mathrm{AC}$ of $0.065 \mu \mathrm{Ci} / \mathrm{cc}$ at the time of the first scan and were scanned multiple times while they decayed to $0.007 \mu \mathrm{Ci} / \mathrm{cc}$. It was subsequently decided that the error in phantoms with higher backgrounds should be evaluated, so one additional phantom of each thickness was made with an initial background of $0.5 \mu \mathrm{Ci} / \mathrm{cc}$ and scanned multiple times until they had decayed to $0.065 \mu \mathrm{Ci} / \mathrm{cc}$. The actual background activity concentrations at the times of the scans were $0.5,0.25,0.125,0.065,0.044,0.032,0.010$ 
and $0.007 \mu \mathrm{Ci} / \mathrm{cc}$. A total of 190 scans were acquired for this specific aim, including the 30 scans acquired to evaluate the total quantitative error.

\section{Calculation of Error}

The error in the background and lesions was calculated as described for Specific Aim 1 for each phantom at every background activity concentration. The error at the lowest concentration $(0.007 \mu \mathrm{Ci} / \mathrm{cc})$ was assumed to have minimal contribution from count rate effects and was used for comparison with the error at higher activities. The difference in error in each phantom at high background $\mathrm{AC}$ and $0.007 \mu \mathrm{Ci} / \mathrm{cc}$ background AC was assumed to be due to count rate effects at each activity level. A surface plot was generated showing how the error due to count rate varies with background $\mathrm{AC}$ and phantom thickness. Additionally, the error due to count rate in lesions was plotted for phantoms with $0.065 \mu \mathrm{Ci} / \mathrm{cc}$ background $\mathrm{AC}$.

To validate the results obtained with phantoms, an additional experiment was performed whereby the effect of count rate was evaluated in the absence of attenuation and scatter. A thin film of gelatin was prepared with 18 F-FDG in a 15-cm diameter Petri dish and scanned multiple times while it decayed. The gelatin was centered in the FOV halfway between the paddles which were separated by $4 \mathrm{~cm}$ for each scan. The initial activity concentration $(6.8 \mu \mathrm{Ci} / \mathrm{cc})$ was chosen to yield the same count rate as was observed in the 4-cm phantoms with $0.065 \mu \mathrm{Ci} / \mathrm{cc}$ background. During each subsequent scan, the count rate from the film was equal to the count rate during one of the scans of the 4-cm phantoms. A large ROI was drawn in images of the film and the mean pixel values at each count rate were normalized to the mean pixel value measured at the lowest 
count rate. For comparison, the mean pixel values in the background of 4-cm phantoms at each activity concentration were also normalized to the mean in the phantoms at the lowest activity. The relative signals of the film and phantoms were plotted against count rate and compared. The uncertainty of the film measurements was estimated from the standard deviations of pixel values in each ROI. The uncertainty of the phantom measurements was estimated from the standard deviations of the mean ROI measurements in the phantoms.

\section{Specific Aim 3: Error due to Attenuation}

One of the most important corrections for accurate quantitation with PET is for attenuation. Prior to hybrid PET/CT scanners, coincidence transmission scans were commonly used for this purpose. The technique uses two sinograms which are acquired while a positron-emitting rod source (e.g. Germanium-68) is rotated around the FOV (62). A reference, or blank, sinogram is acquired with nothing in the FOV and a transmission sinogram is acquired with the patient in the FOV. Only coincidence events which are collinear with the known location of the line source are counted in either scan because all others must be due to randoms or scatter. The attenuation along each LOR is calculated by comparing the count rates in the transmission sinogram to the count rates in the reference sinogram. A similar approach was used to measure the error due to attenuation on the PEM Flex system. However, because the goal of this work was to measure the error in images, measurements were based on reconstructed images rather than projection data. 


\section{Point Source Transmission Scans}

Blank and transmission scans of a point source were acquired to evaluate the error due to attenuation in gelatin breast phantoms of multiple sizes. A low activity point source was rigidly attached to the center of the bottom paddle and scanned with cold (non-radioactive) gelatin stacked on top of it and again with nothing in the FOV. It was assumed that the differences in the maximum pixel values in images acquired in gel and in air at the same paddle separation were primarily due to attenuation and source activity, as argued in the Discussion. The maximum pixel values were normalized to activity and compared to calculate the error due to attenuation.

A point source of approximately $20 \mu \mathrm{Ci}$ was centered on the bottom paddle of the PEM Flex scanner and taped in place (Figure 2.2). The low activity was chosen to minimize dead time and randoms. Even though the point source was smaller than a pixel $\left(1.2 \times 1.2 \times 1.2 \mathrm{~mm}^{3}\right)$, partial volume effects $(\mathrm{PVE})$ were not considered important because only relative measurements were being made. Further, it was assumed that PVE would be the same for scans acquired with the same detector separation as long as the source did not move with respect to the paddles between scans.

A series of scans was acquired with cold gelatin breast phantoms of different thicknesses assembled on top of the source. The phantom scans were acquired in order of decreasing thickness $(12,10,8,6$ and $4 \mathrm{~cm})$ in order to help maintain similar count rates during all scans. Data were acquired for 10 minutes while the detectors translated across the entire FOV with the paddles set to the thickness of each phantom. Air scans were subsequently performed with the detector paddles separated by each of the same distances, in order of decreasing separation. The source was allowed to decay before the 
first air scan to ensure the count rate in air was similar to that in the gel scans. This experiment was performed three times for a total 30 separate scans.

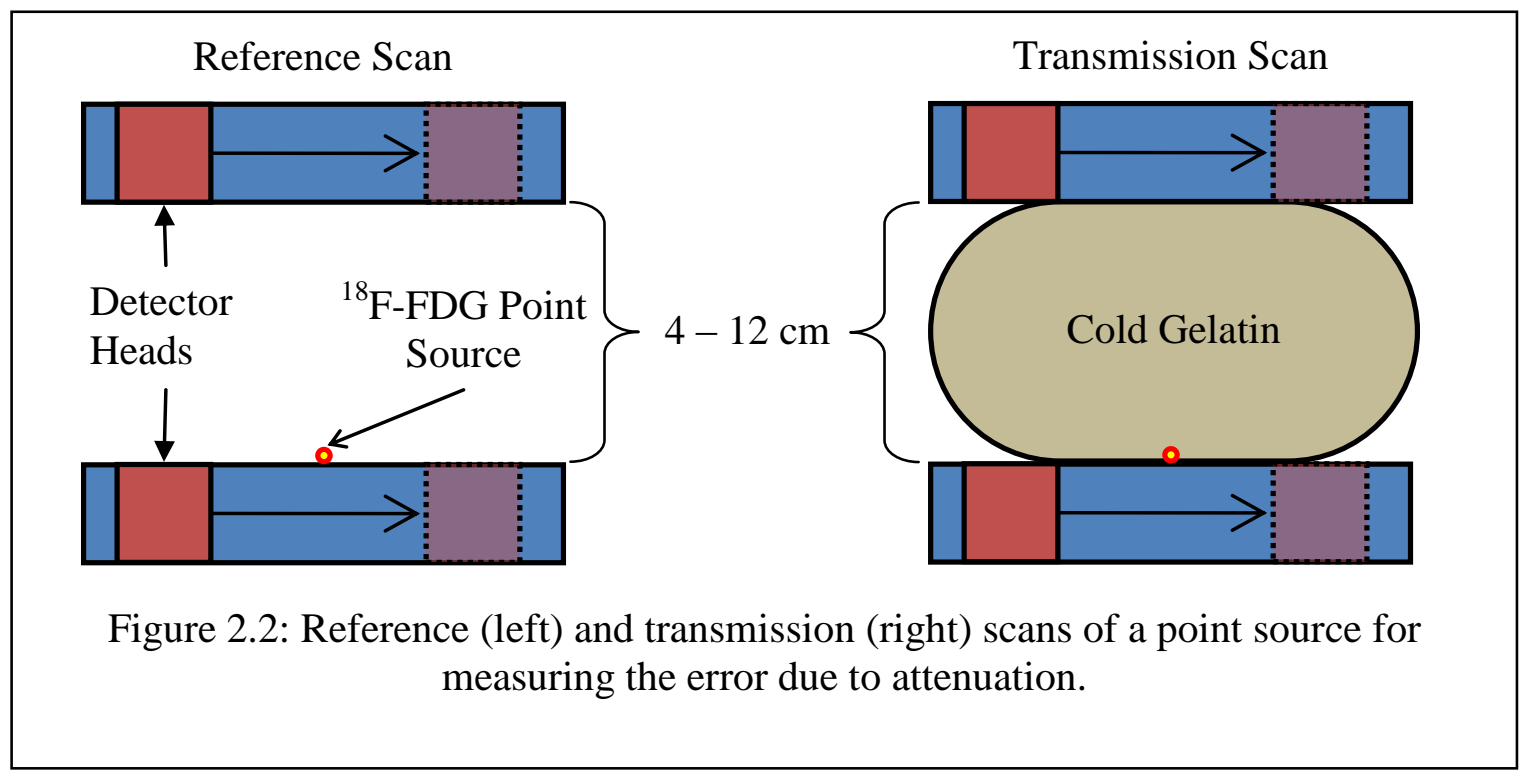

\section{Calculation of Error}

Images were reconstructed with the standard clinical protocol (Table 2.1) and evaluated on the scanner console in units of $\mu \mathrm{Ci} / \mathrm{cc}$. The maximum pixel value in the plane corresponding to the point source was measured for each set of images. The maxima were normalized to the source activity at the time of each scan because images displayed in units of $\mu \mathrm{Ci} / \mathrm{cc}$ are not decay-corrected. For each phantom, the error due to attenuation was calculated from the percent difference was calculated between the normalized maxima in gel and in air at the same paddle separation (Equation 2.3). The results from three experiments were averaged and plotted versus thickness.

$$
\% \text { Error }_{\text {Attenuation }}=100 \times\left(\operatorname{Max}_{\text {Gel }}^{\prime}-\text { Max }_{\text {Air }}\right) / \text { Max }_{\text {Air }}
$$




\section{Specific Aim 4: Error due to Scatter}

A method for evaluating scatter in sinograms (63) was modified and used used to measure the error due to scatter in images. Their setup involved a line source which was scanned in air and in a water-filled phantom. Three sinograms were produced: $g_{\text {air }}$ from the scan in air; $g_{\text {water }}$ from the scan in water; and $g_{a t t e n}$, which was the calculated attenuation introduced by the water for each projection bin. The total counts in the water were found by integrating $g_{\text {water }}$ and the true counts in water were estimated by integrating the product $g_{\text {atten }} \times g_{\text {air }}$. The estimated true counts were subtracted from the total counts in water, the difference being the counts due to scatter. The scatter fraction was the ratio of this difference and the total counts from the water scan (Equation 2.4):

$$
S F=\left[\left(\sum g_{\text {water }}-\Sigma g_{\text {air }} \times g_{\text {atten }}\right) / \Sigma g_{\text {water }}\right]
$$

The method used by Bailey and Meikle was modified and applied to images from the PEM Flex system. First, a point source was used instead of a line source. The source was scanned with and without gelatin phantoms in the FOV. Second, rather than integrating projection data to calculate counts, pixel values were summed to calculate the total activity within the image volumes. The sum of pixel values with gelatin were compared to the sum without, and the error due scatter was estimated.

\section{Point Source Transmission Scans}

The error due to scatter was measured in the same image sets as were used for calculating the attenuation error. To reiterate, a point source was centered on the bottom paddle and scanned with $4-12 \mathrm{~cm}$ of cold gelatin on top of it. The paddles were set to the thickness of each phantom for the scans. Scans without gelatin were acquired at each 
of the same paddle separations.

\section{Calculation of Error}

An ROI was drawn around the entire FOV in one image from each scan and propagated to all slices. The scanner console reports the sum of values in an ROI as the total activity $(\mu \mathrm{Ci}$ ) in the region (the product of activity concentration in $\mu \mathrm{Ci} / \mathrm{cc}$ and $\mathrm{ROI}$ volume in $\mathrm{cm}^{3}$ ). The total activity measured in each image volume was normalized to the actual activity of the source at the time of each scan. The total activity (or signal) in gelatin was assumed to differ from that in air for two primary reasons: attenuation and scatter. The signal expected due to attenuation was estimated by artificially attenuating the total activity measured in air at the same paddle separation. While Bailey and Meikle calculated the attenuation from the path length of water in each bin of their water sinograms, the effective attenuation for this work was taken from the results from the attenuation measurements described in Specific Aim 3. The signal due to scatter was calculated by taking the difference between the total signal in gelatin and the estimated signal due to attenuation at the same thickness. The error due to scatter was determined from the ratio of the signal due to scatter and the total activity in air (Equation 2.5),

$$
\% \text { Error }_{\text {Scatter }}=100 \times\left(\text { Total }_{\text {Gel }}-\text { Total }_{\text {Air }} \times \text { Attenuation }\right) / \text { Total }_{\text {Air }}
$$

where Total $_{G e l}$ is the total activity measured in gelatin, Total $_{A i r}$ is the total activity measured in air and Attenuation is the relative signal expected due to attenuation. 


\section{CHAPTER 3: RESULTS}

\section{Specific Aim 1: Total Quantitative Error}

The total quantitative error in the uniform background and embedded lesions in 4 $-12 \mathrm{~cm}$ thick gelatin breast phantoms is plotted in Figure 3.1 where the error bars indicate 1 standard deviation of three measurements. The error in the uniform background was negative for virtually all thicknesses, with the exception of the 4-cm thick phantom in which the average background error was $0 \pm 7 \%$. The background error increased (i.e., became more negative) with thickness.

The error in lesions was negative for all thicknesses and LBRs and it was much greater than the background error. As in the background, the error in the lesions increased with phantom thickness and it also increased non-linearly with LBR. The contrast error of the cysts was greater contrast than the error in any of the lesions.

PEM images from gelatin breast phantoms of each thickness are displayed in Figure 3.2 with the same window width and level. The signal in the background, lesions and cysts decreased with thickness, as well as the contrast of the lesions and cysts. A broad band of enhancement is visible along the chest wall edge of the FOV (top) in the 4 $\mathrm{cm}$ phantoms and it decreases in severity with phantom thickness. This artifact is also visible but much less conspicuous in clinical images of breast compressed to $6 \mathrm{~cm}$ or less. The manufacturer is aware of the artifact and does not have an explanation or a correction for it. The 5:1 and 10:1 lesions in a uniform background of $0.065 \mu \mathrm{Ci} / \mathrm{cc}$ are visible in all 12 images from one 12-cm thick phantom (Figure 3.3). The pixels near the edges of the 
FOV are very noisy compared to the central FOV, particularly the first row of pixels along the chest wall edge (Figures 3.2 and 3.3).
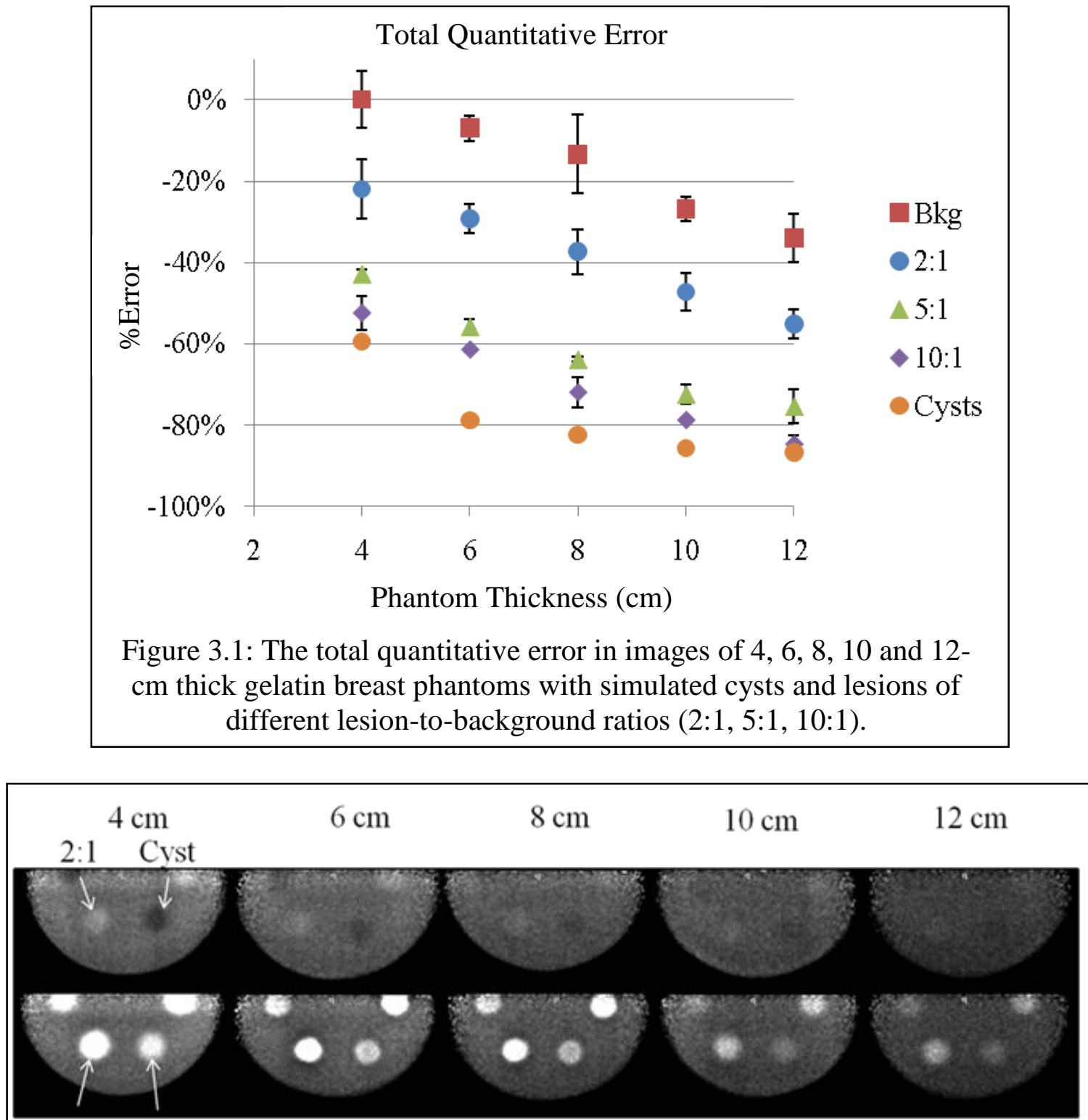

\section{$10: 1 \quad 5: 1$}

Figure 3.2: Images of lesions and cysts in gelatin breast phantoms of each thickness with $0.065 \mu \mathrm{Ci} / \mathrm{cc}$ background $\mathrm{AC}$. Images are displayed with the same window width and level $(14,000 / 7,000 \mathrm{~Bq} / \mathrm{ml})$. 


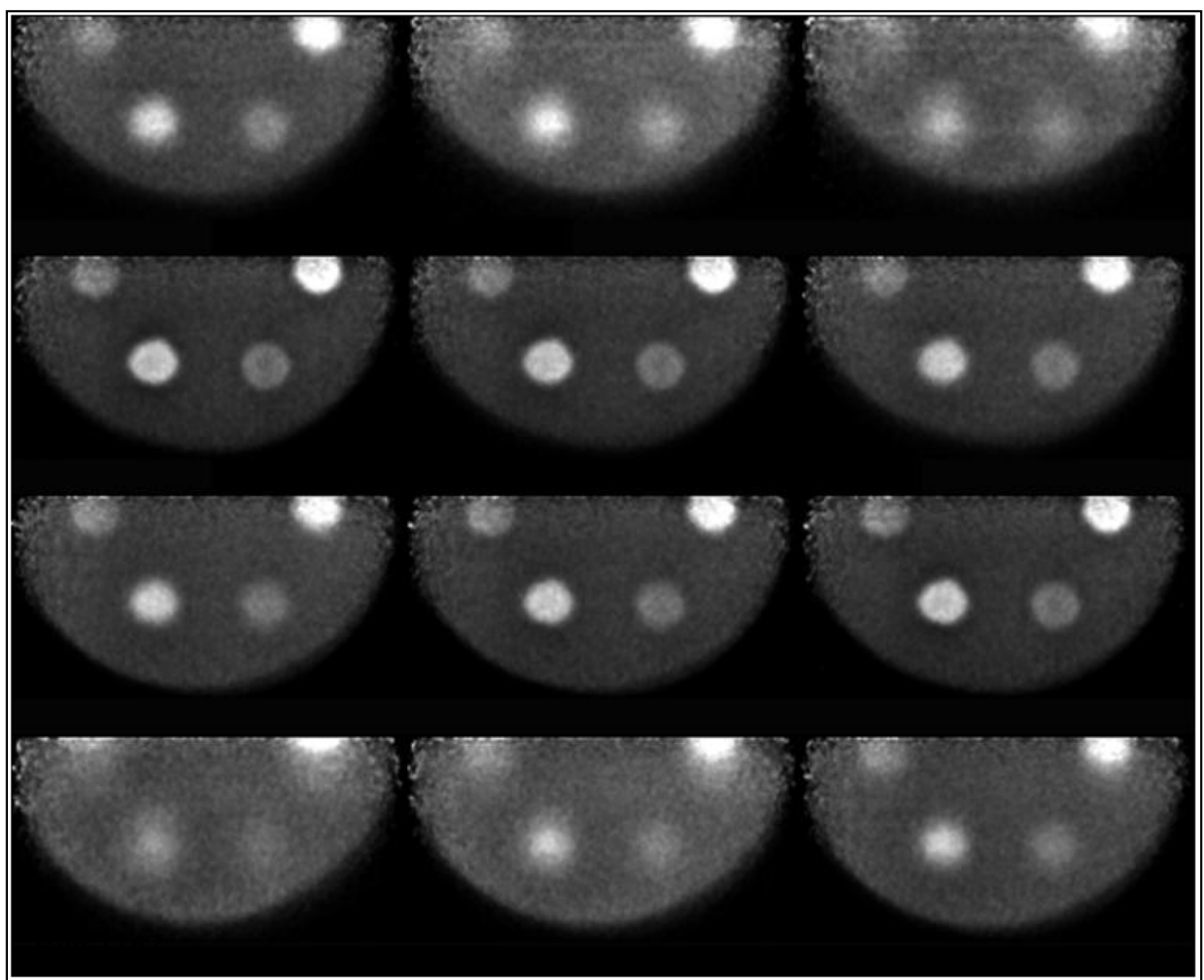

Figure 3.3: All 12 PEM images (1-cm thick) of a 12-cm thick phantom with 5:1 and 10:1 lesions in a uniform background $\mathrm{AC}$ of $0.065 \mu \mathrm{Ci} / \mathrm{cc}$.

\section{Specific Aim 2: Error due to Count Rate}

A surface plot of the count rate error in the uniform background (Figure 3.4) shows that the count rate contributed large negative errors for virtually all activity levels evaluated. The error due to count rate increased with background activity concentration, and decreased with thickness. 


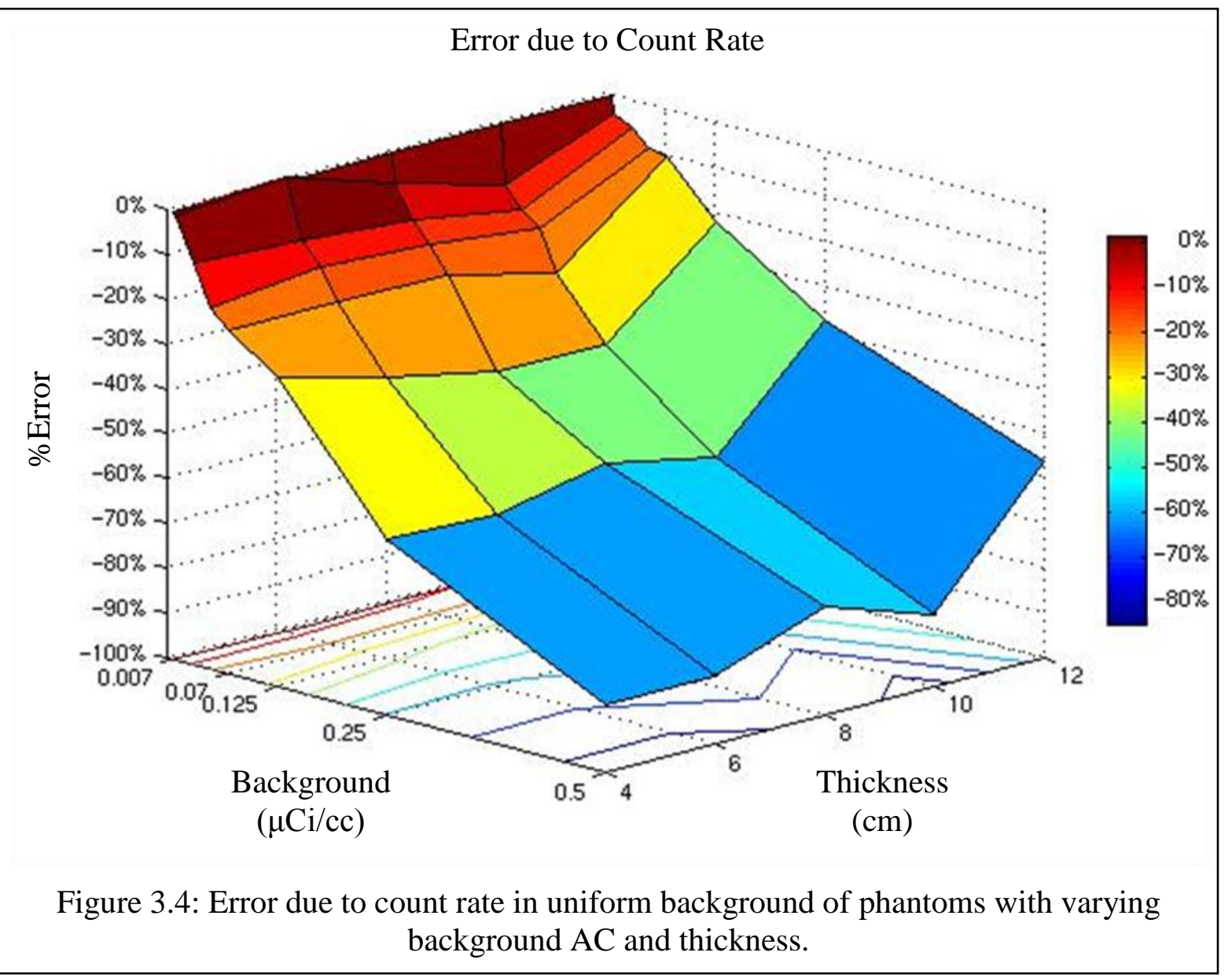

The count rate error in lesions of the phantoms with $0.065 \mu \mathrm{Ci} / \mathrm{cc}$ background is also plotted for comparison with the count rate error in the uniform background (Figure 3.5). At this activity the 2:1 lesions had more error due to count rate than the background for some phantom thicknesses but it did not change monotonically. The count rate error in lesions decreased with respect to thickness in the 5:1, and remained relatively constant with thickness in the 10:1 lesions. The count rate error in lesions decreased non-linearly with LBR for all thicknesses.

The mean signal in images of a thin film of gelatin scanned multiple times as it decayed was normalized to the signal at the lowest activity and plotted as a function of count rate. The mean signal in the background of 4-cm phantoms was also normalized to 
the signal at the lowest activity and plotted. The normalized signal in the film follows the same trend with count rate as the normalized signal in 4-cm phantoms.

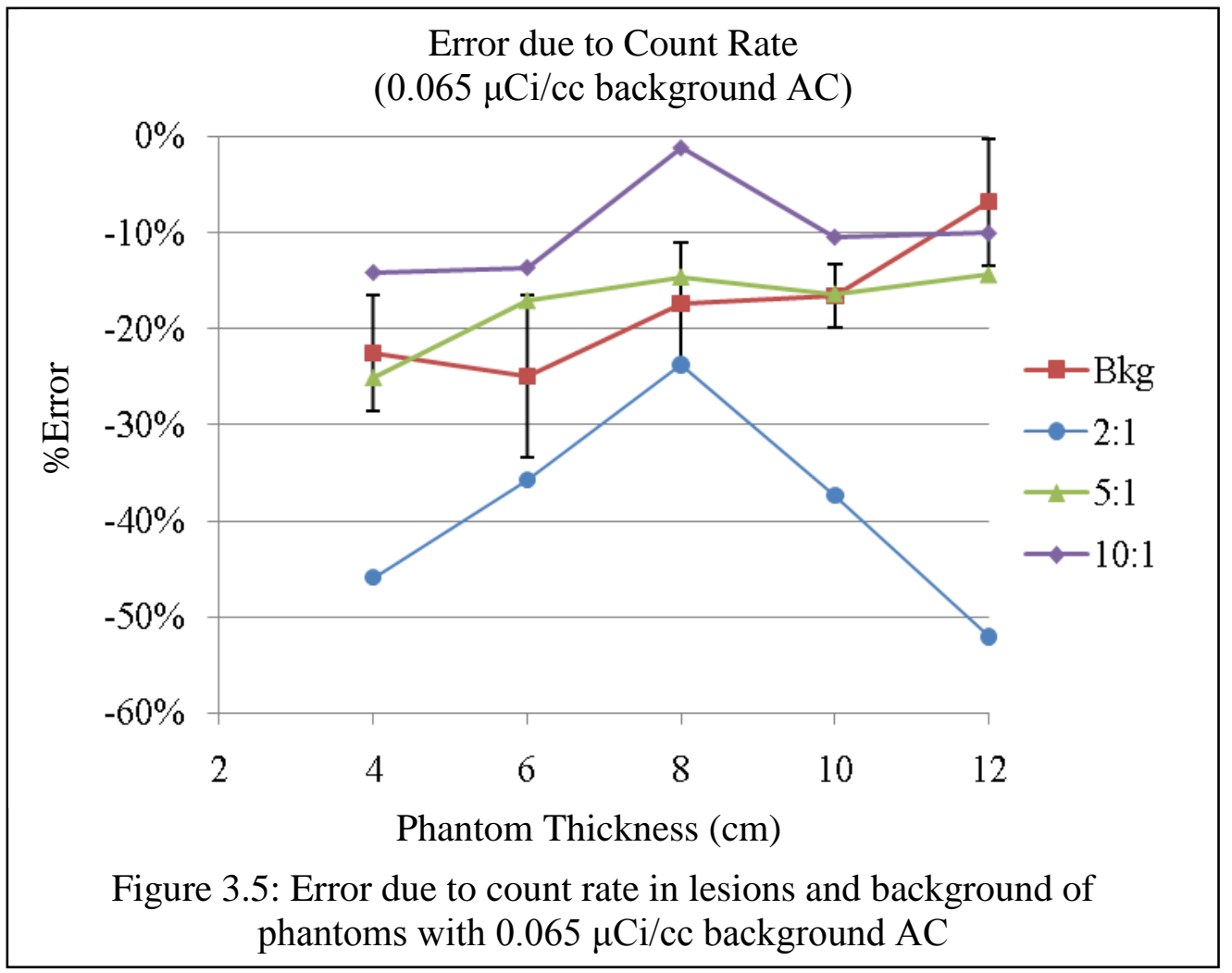

\section{Specific Aim 3: Error due to Attenuation}

The error due to attenuation is plotted in Figure 3.6 with error bars indicating the standard deviation of three measurements. For comparison, the black curve is the percentage of signal loss expected due to narrow-beam attenuation of $511 \mathrm{keV}$ photons in water $\left(\mu=0.096 \mathrm{~cm}^{-1}\right)$.

The error due to attenuation was negative and large for all thicknesses, and it increased with thickness (from $-51 \pm 10 \%$ to $-77 \pm 4 \%$ at 4 and $12 \mathrm{~cm}$, respectively). The measured error was greater than the relative signal loss calculated for narrow-beam attenuation. 


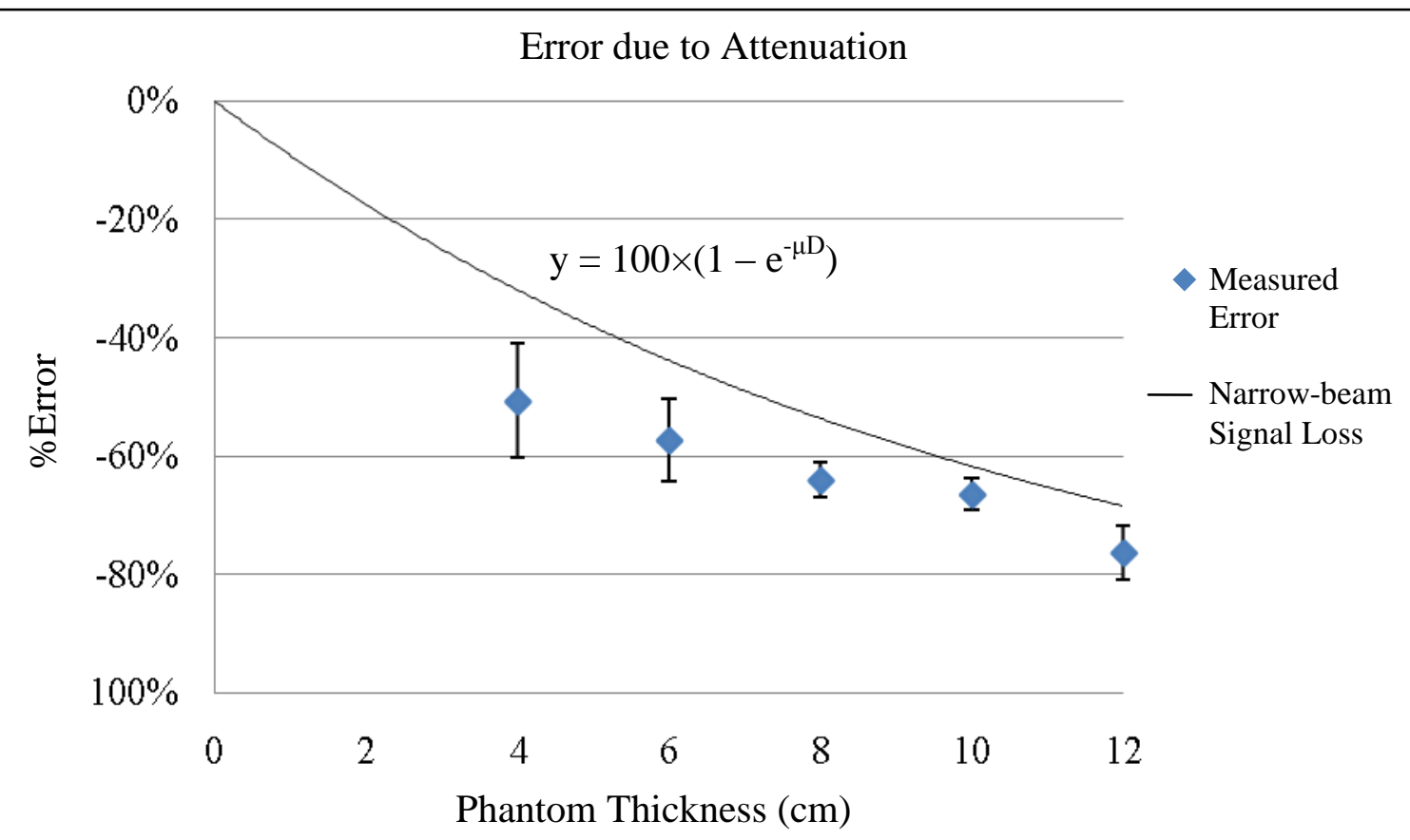

Figure 3.6: The measured error due to attenuation with the percent signal loss expected due to attenuation of $511 \mathrm{keV}$ photons in the given thicknesses (D) of water.

\section{Specific Aim 4:Error due to Scatter}

The total activity measured in gelatin was greater than what would be measured from attenuated true LORs alone. The additional activity due to scatter was found to be a relatively constant fraction of the total activity in air (Figure 3.7). 


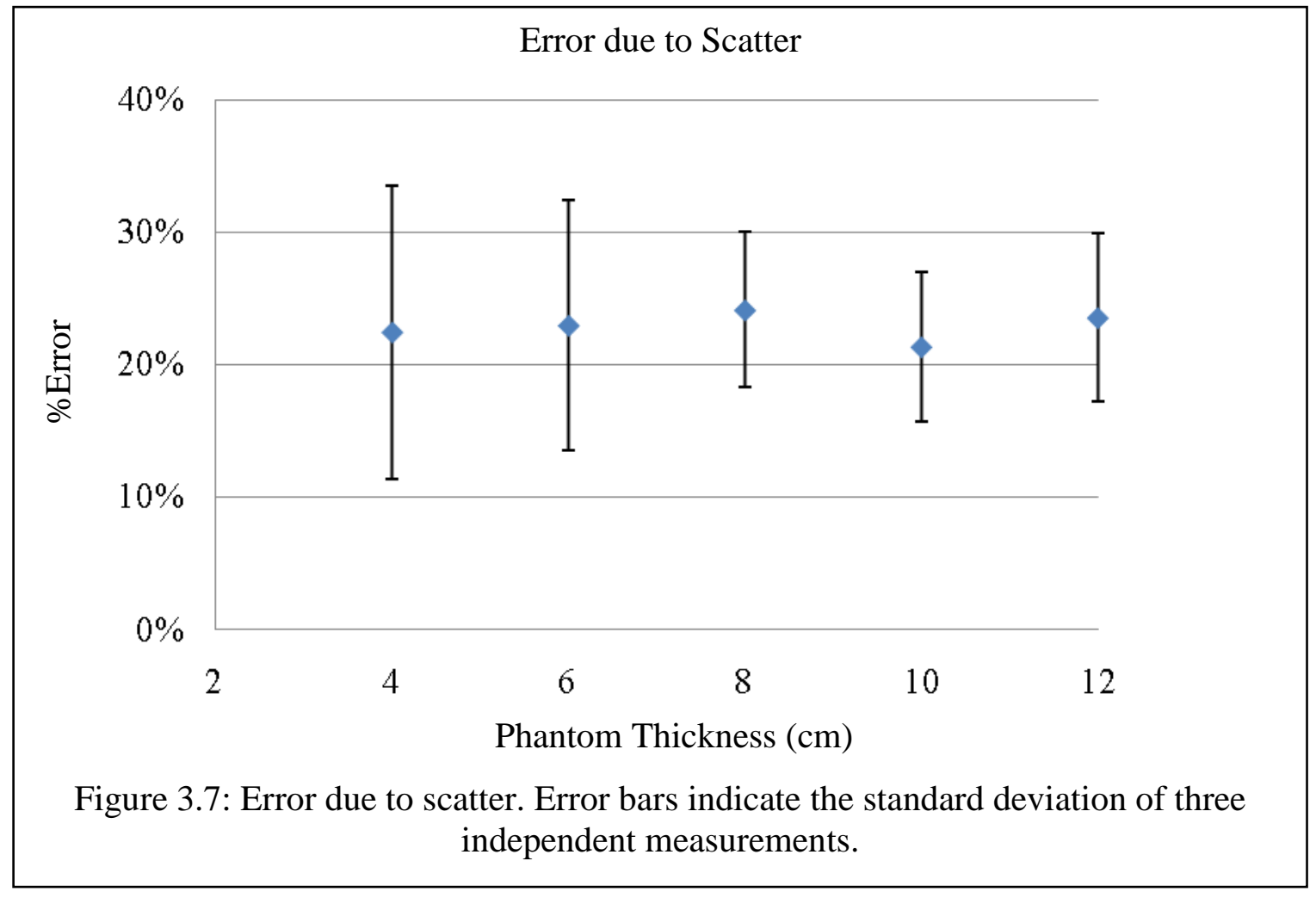




\section{CHAPTER 4 - DISCUSSION AND CONCLUSIONS}

As is true for conventional PET, PEM has many competing sources of quantitative error including, but not limited to, count rate effects (e.g. dead-time, random coincidences, pulse pile-up), attenuation and scattered photons. The results suggest additional sources of error.

\section{Specific Aim 1: Total Quantitative Error}

The competitive nature of the individual errors resulted in good agreement $(0 \pm 7 \%$ error) between the measured and known activity concentration in the background of 4-cm phantoms (Figure 3.1). The background error in thicker phantoms was negative and increased with thickness (Figure 3.1), as is visually evident in Figure 3.2. This trend is consistent with the fact that signal decreases due to attenuation with increasing thickness, thus contributing negatively to the total error. Attenuation is not corrected for on the PEM Flex Solo II and its impact on images is evaluated in Specific Aim 3.

The error in the lesions of all LBRs is greater than the error in the background and it increases with LBR. This behavior indicates an additional source of quantitative error which is not apparent in the uniform background: limited-angle tomography (LAT). The effect of LAT on quantitation has been calculated by Murthy, Aznar, Thompson, Loutfi, Lisbona and Gagnon (64) and their formulation (Equation 4.1) can be rearranged to show that the error in measured lesion $\mathrm{AC}$ is inversely proportional to LBR.

$$
L B R_{\text {Measured }}=L B R_{\text {True }} \frac{d}{D}+\frac{D-d}{D}
$$

where $L B R_{\text {Measured }}$ is the measured $\mathrm{LBR}, L B R_{\text {True }}$ is the true LBR, $d$ is the lesion 
dimension perpendicular to the detectors and $D$ is the phantom thickness.

In LAT, many projection angles are missing and cannot be used to constrain activity along existing LORs. Thus, LAT mispositions signal along LORs, essentially smearing objects in images perpendicularly to the detectors. The signal lost from lesions, which is represented by the first term in Equation 4.1, is misplaced in other planes, making the lesions visible throughout most or all of the other image planes (Figure 3.3). This loss of signal from the lesions explains why the error is greater in lesions than in the background. Another interesting aspect of the lesion error is that it increases non-linearly with. This behavior of the lesion error is also partially attributable to LAT, because while much signal is removed from the lesions, some signal is added back from spill-over, as well as from scatter and randoms. The second term in Equation 4.1 represents the effect of spillover. Similarly, the signal in the cysts is due to mispositioned signal from spillover, in addition to scatter and randoms, which explains the loss of contrast.

The breast phantom images in Figure 3.2 visually demonstrate a loss of signal in the background, lesions and cysts, consistent with the increasingly negative error shown in Figure 3.1. Additionally, the images exhibited artifacts which could further affect the quantitative accuracy. First, the bright band along the chest wall edge (top) of the FOV (Figure 3.2) caused measurements there to be higher than farther inside the FOV. These measurements were excluded from the results reported here. This artifact is likely due to different dead-time in the detector modules along that edge compared to modules farther inside the FOV. The count rate was probably lower in those modules for two reasons. First, there was no activity outside the FOV, so there were fewer single events to induce dead time. However, this artifact is visible in clinical images of patients' breasts even 
though there is activity in normal tissues outside the FOV during patient scans. Second, to a lower singles rate, detector modules near the edge of the FOV might experience lower coincidence processing time. The modules near the edge of the FOV will detect photons in coincidence with fewer modules in the opposing detector than will the modules near the center. With fewer coincident prompts and decreased coincidence processing time, the modules near the edges detect a greater fraction of LORs between modules at the edge of the FOV, hence greater signal in this region.

Another artifact is the noise around the edges of the FOV. Relatively few events can be detected along LORs in these two regions for different reasons. Along the top edge, LORs which contribute signal to the topmost row of pixels are confined to a single plane perpendicular to the detectors. This reduces the number of events which can contribute to a voxel in that plane and increases the noise, which is amplified by correcting for geometric efficiency. Near the left and right ends of the phantom, LORs are not constrained to a single plane, rather the translation of the detectors results in less time spent collecting data along each LOR. Fewer events in these two regions result in lower sampling statistics than farther inside the FOV, hence greater noise.

\section{Specific Aim 2: Error due to Count Rate}

The phantoms used to measure the overall quantitative error were scanned multiple times while they decayed from $0.05 \mu \mathrm{Ci} / \mathrm{cc}$ to $0.007 \mu \mathrm{Ci} / \mathrm{cc}$. The background error at each background activity was compared to the error with the lowest background and the difference was attributed to the error introduced by count rate.

The negative error contributed by the count rate (Figure 3.4) is primarily due to 
increased dead time, which reduces the fraction of all events which are counted. The fact that the count rate error decreases with thickness is easily explained by another source of error, attenuation. The greater total activity in thicker phantoms was more than compensated for by exponential attenuation, which resulted in fewer total events being collected in thicker phantoms. Thus, dead time and error due to count rate decrease with phantom thickness.

It is interesting that the error due to count rate in lesions is different than in the background. This could be due in part to the fact that the count rate varies as the detectors pass of the lesions, although the error due to count rate would be expected to increase over the lesions due to the higher count rates. The opposite was observed which might actually be another result of LAT. Murthy's formulation (Equation 4.1) again can be used to predict that the count rate error in lesions is inversely proportional to LBR. The reason is that at least some of the signal in lesions is due to spillover from the background, and by extension the error in the lesions depends on the error in the background. A change in the background error due to count rate will have a relatively smaller effect on the lesions.

\section{Specific Aim 3: Error due to Attenuation}

The maximum pixel values in images of a point source scanned in air and in gelatin were used to measure the error due to attenuation, similarly to blank and transmission scans used for attenuation correction of conventional PET scans. The argument for using the maximum pixel values is as follows. Blurring due to noncollinearity is on the order of $0.22 \%$ of the detector separation (Cherry, Sorenson, Phelps 2003), which is smaller than the point source $\left(\sim 1 \mathrm{~mm}^{3}\right)$, even for the largest paddle 
separation on the PEM Flex scanner ( $0.44 \mathrm{~mm}$ blurring with $20 \mathrm{~cm}$ paddle separation). Therefore, all true LORs can be assumed to intersect the point source. Because the point source was smaller than a pixel $\left(1.2 \times 1.2 \times 1.2 \mathrm{~mm}^{3}\right)$, the maximum pixel value corresponded to the approximate location of each source. Scattered photons, by definition, will necessarily result in misplaced LORs. Only small angle scatter, which comprises a small fraction of scatter, will contribute signal to the maximum pixel value. Randoms can be assumed to be negligible at sufficiently low count rates so essentially no random LORs will intersect the maximum pixel value. Thus, the maximum pixel value would contain signal primarily due to true LORs, with minimal signal from scatter and no signal due to randoms. Comparing the decay-corrected maximum pixel value of the point source in gelatin to that in air is a valid way to estimate the error due to attenuation.

The error due to attenuation was negative and increased with thickness (Figure 3.5), as expected. However, the magnitude of the error was greater than the relative signal loss expected due to narrow beam attenuation of $511 \mathrm{keV}$ photons by water (Figure 3.5). This is due to the attenuation of signal from oblique LORs, which experience longer path lengths in the phantoms and thus more attenuation. The attenuation along the most oblique LORs was calculated for each thickness, as described in Figure 4.1, and the relative signal loss was plotted along with the measured data (Figure 4.2). The results indicate that the effect attenuation has on the most oblique LORs is greater than the measured error. This is consistent because the LORs acquired have a distribution of angles, and most are between the direct and the most oblique LORs. 


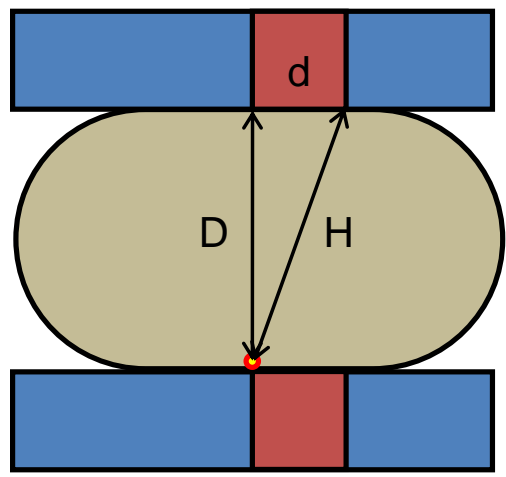

Detector width: $\mathrm{d}=5.5 \mathrm{~cm}$

Signal along direct LORs: $\mathrm{S}(\mathrm{D})=\mathrm{S}_{0} \mathrm{e}^{-\mu \mathrm{D}}$

Max path length: $H=\left(D^{2}+d^{\prime 2}\right)^{1 / 2} ; d^{\prime}=2^{1 / 2} d$

Signal along max path length:

$$
\mathrm{S}(\mathrm{H})=\mathrm{S}_{0} \mathrm{e}^{-\mu \mathrm{H}}=\mathrm{S}_{0} \exp \left[-\mu\left(\mathrm{D}^{2}+2 \mathrm{~d}^{2}\right)^{1 / 2}\right]
$$

Figure 4.1: Path length and attenuation of oblique LORs. Both depend on angle of incidence. The maximum path length is the hypotenuse $(\mathrm{H})$ of the right triangle with one side equal to the detector separation (D) and the base (d') equivalent to a crystal separation of 25 in the $\mathrm{x}$ - and $\mathrm{y}$-directions.

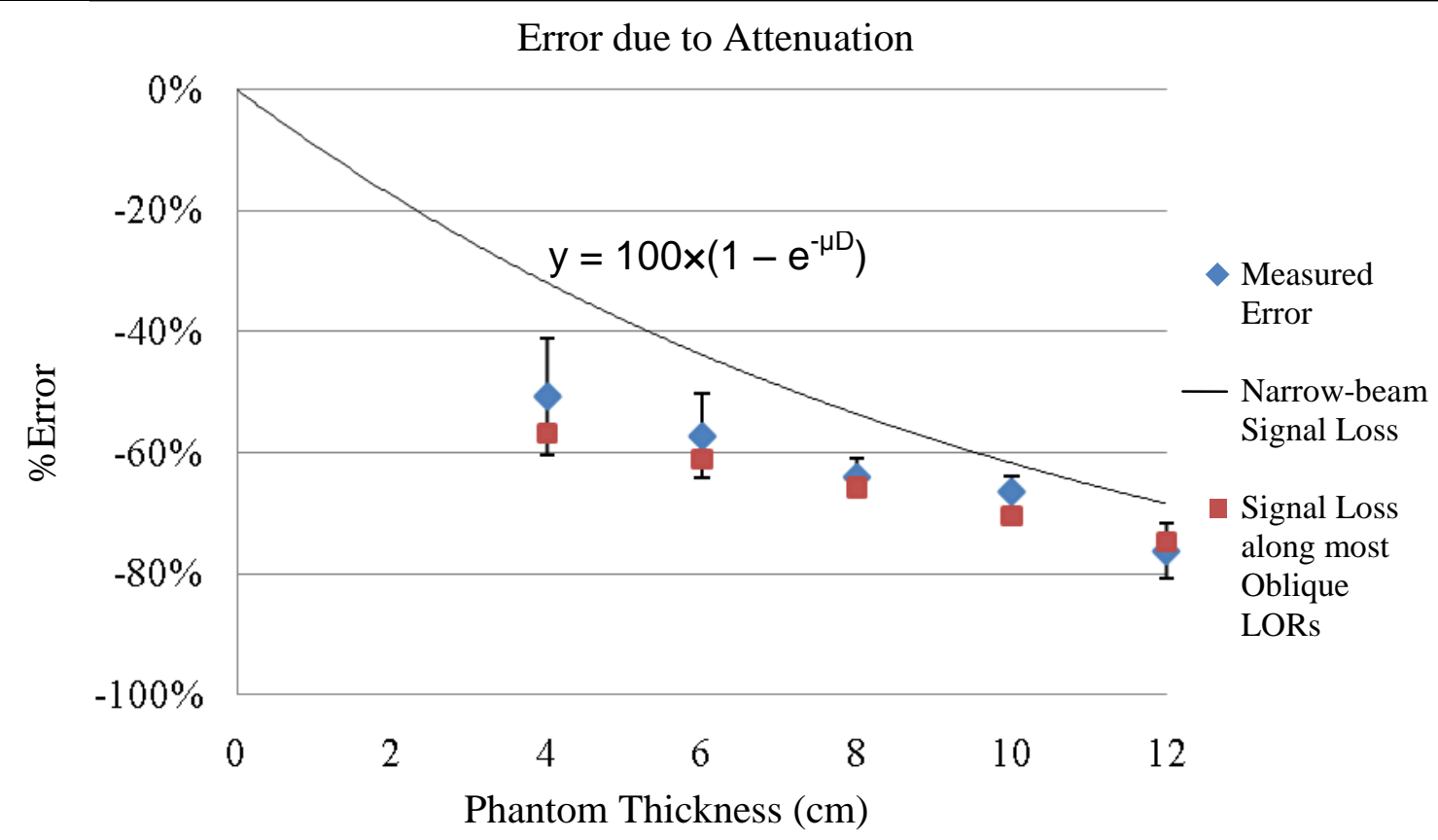

Figure 4.2: The signal loss due to attenuation along the most oblique LORs. Also plotted are the percent signal loss expected due to attenuation of $511 \mathrm{keV}$ photons in the given thicknesses $(D)$ of water, and the measured error due to attenuation. 


\section{Specific Aim 4: Error due to Scatter}

The error due to scatter was investigated with images of a point source scanned with and without cold gelatin breast phantoms on top of it. The total signal in the reconstructed image volumes was measured and the results from Specific Aim 3 were used to estimate the signals due to attenuation and scatter, from which the error due to scatter was calculated.

Scatter introduced a somewhat large $(23 \%)$ positive error, which is expected. What may seem counterintuitive is that the error due to scatter was relatively independent of phantom thickness. This may be unexpected because, as is well known, the scatter fraction in conventional PET increases with patient size (59). The results are actually consistent with this fact because the total signal from larger phantoms or patients decreases due to attenuation. Thus, a constant signal due to scatter (which would yield a constant error) would represent a greater fraction of the total signal.

The reasons scatter contributes a relatively constant signal with phantom thickness may not be immediately obvious, but it can be explained from first principles, as illustrated in Figure 4.3. Thicker phantoms (and patients) scatter a greater number of photons, according to Equation 4.2.

$$
S=1-\exp (-\sigma D)
$$

where $S$ is the total fraction of photons which are scattered, $\sigma$ is the Compton scatter coefficient $\left(0.0956 \mathrm{~cm}^{-1}\right)$, and $D$ is the phantom thickness. If all of the scattered photons were measured, the signal due to scatter would actually increase. This is not the case, however, because the scattered photons are themselves attenuated. If one assumes for the sake of argument that scattered photons experience the same attenuation as 511 
$\mathrm{keV}$ photons, then the net signal measured from scatter (Equation 4.3) can be approximated from the product of Equation 4.2 and the exponential attenuation for the given thickness.

$$
S^{\prime}=\exp (-\mu D)[1-\exp (-\sigma D)]
$$

where $S^{\prime}$ is the net signal due to scattered photons which undergo attenuation, and $\mu$ is the linear attenuation coefficient of $511 \mathrm{keV}$ photons in water $\left(0.0959 \mathrm{~cm}^{-1}\right)$. Equation 4.3 overestimates the net signal due to scatter because photons lose energy when scattered (65). Thus, the linear attenuation coefficient of scattered photons is higher than $511 \mathrm{keV}$ photons (17\% higher for the lower level discriminator, $350 \mathrm{keV})$. Nevertheless, the plot of Equation 4.3 in Figure 4.3 shows that this approximation exhibits the behavior observed. Specifically, the net signal due to scatter does not change monotonically between 4 and $12-\mathrm{cm}$ of water. This approximation is also consistent with the measurements of Watson, Case, Bendriem, Carney, Townsend, Eberl, Meikle and Difilippo, (59) who showed that counts due to scatter actually decrease with patient mass, as is indicated in the figure for thicknesses corresponding to the sizes of human patients $(>24 \mathrm{~cm})$. 


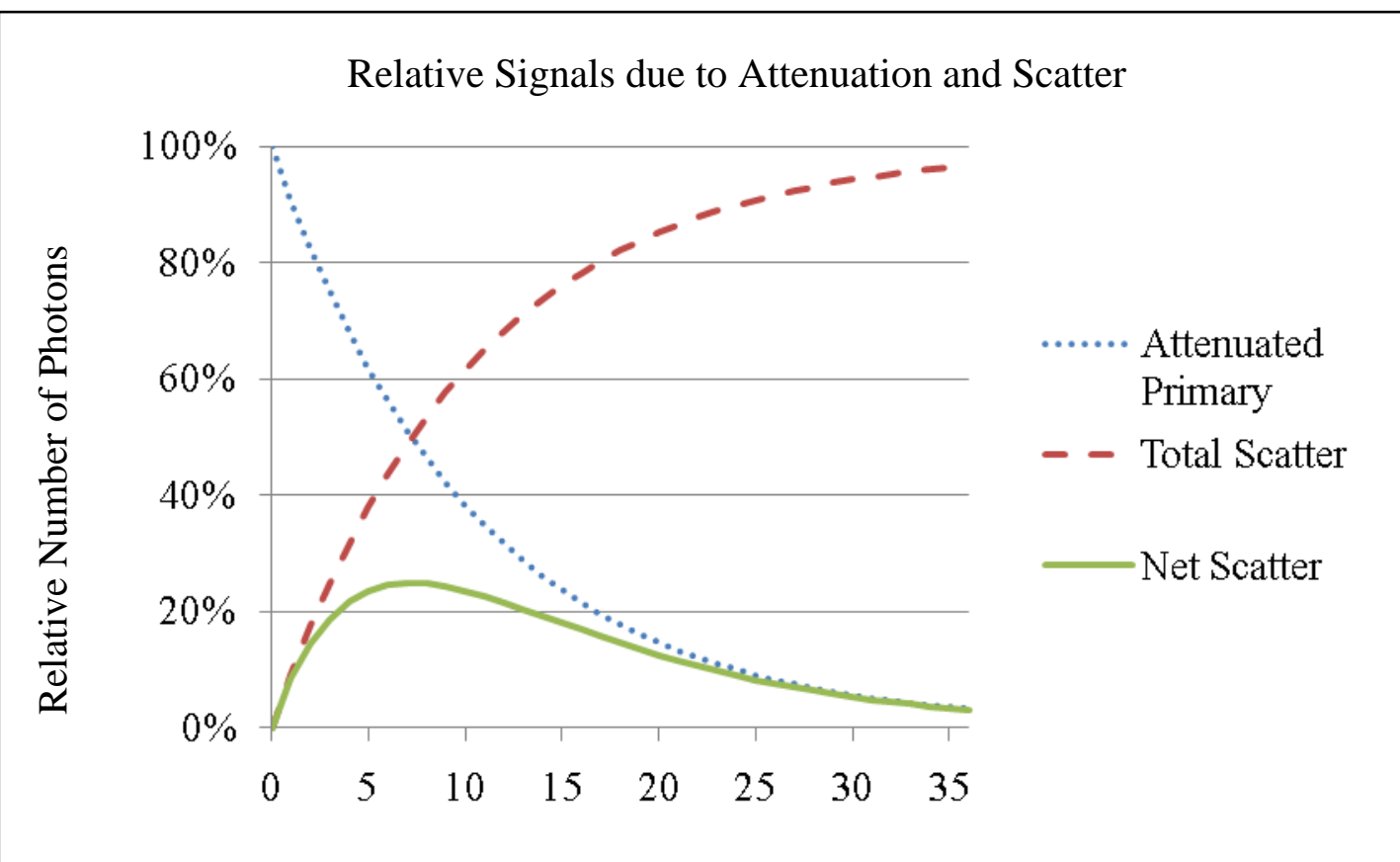

Phantom Thickness $(\mathrm{cm})$

Figure 4.3: Signals due to attenuation and scatter

The sum of these measured sources of error is not equal to the total error measured for any thickness, in the lesions or background, suggesting that other sources of error exist. For example, the effect of LAT was observed in lesions and cysts but it was not quantified by this work. Another source of error may be detector normalization. The relative efficiency of a crystal pair is affected, in part, by the effective area of the crystals and the maximum path lengths photons can travel in them, as well as shielding by intervening crystals. All of these factors depend on crystal separation and paddle separation, however data for detector normalization of the PEM Flex system are acquired at only one paddle separation $(15 \mathrm{~mm})$. Thus, the normalization performed may not be accurate at paddle separations other than $15 \mathrm{~mm}$. 


\section{Limitations}

There are some limitations to these experiments. Perhaps the most significant is the lack of activity outside the FOV for evaluation of the total quantitative error and count rate. Uptake in normal tissue would contribute many singles events, leading to increased randoms rate, as well as some scatter. This effect has been shown to decrease lesion contrast in other PEM systems (66) by increasing the background signal, particularly close to the chest wall edge of the FOV. The effect of activity outside the FOV on quantitation would probably be to artificially increase the signal due to randoms and scatter. Considering the large negative errors measured, this would have artificially reduced the error measured in most cases, and perhaps resulted in positive total error in the background of 4-cm phantoms with $0.065 \mu \mathrm{Ci} / \mathrm{cc}$. By omitting activity outside the FOV, dead time was the dominant count rate effect which contributed to the error.

This work used lesions of one size which were confined to one height between the detectors. However, the measured AC has been shown to vary with lesion size in addition to breast thickness $(44,45,53)$. LAT affects the error in lesions of all sizes (67) while partial volume effects (PVE) are known to contribute to the error in small lesions (45). It has also been shown that the mean and maximum pixel values in identical spheres are higher near the detector face and near the chest wall edge of the FOV (45). In this work differences in background, lesion and cyst measurements were observed near the chest wall edge of the FOV; however, these measurements were not included in the analysis. Future investigations into the absolute quantitative accuracy of the PEM Flex system should include lesions of multiple sizes, at different heights between the detectors and at various distances from the edges of the FOV. 
It is impossible to acquire images without some dead-time, so the error measured due to count rate may have been affected by dead-time in the reference images. Perhaps a way to compensate for this problem would be to extrapolate the total error in low activity phantoms to a background AC. Alternatively, Monte Carlo simulations could calculate the errors contributed by all count rate effects (dead-time, randoms and pulse pile-up.)

Since position has an impact on measurements, the location of the point source used to evaluate attenuation and scatter may also have biased the results. Ideally, scans could be performed with a source positioned at an arbitrary height between the detectors to test this. If that were done, however, it would be impossible to acquire gel scans and air scans without disturbing the source. Due to the size of the point source compared to a pixel, partial volume effects would change if the source moved between scans, introducing a great deal of uncertainty. Also regarding the attenuation measurements, small-angle scatter must contribute some signal to the pixel containing the point source. This contribution is expected to be small, but Monte Carlo simulations may be useful to validate this assumption and corroborate these results.

It was assumed that the maximum pixel value was primarily affected by attenuation, and that scatter contributed signal everywhere else in the image volumes. In reality, some small angle scatter certainly contributed to the maximum pixel value, potentially biasing the measurements of attenuation which were used in calculating the error due to scatter. However, the results of the attenuation experiments were consistent with what should be expected for oblique LORs, lending support to this assumption. As with the attenuation measurements, the amount of scatter measured might have varied if the source were at different heights between the detectors. Such measurements would be 
ideal, but removing the gelatin without disturbing the source would not be practical. If the source were to move between corresponding gelatin and air scans, differences in PVE could have a large impact on the attenuation measurements. Rigidly fixing the point source in place with tape on the bottom paddles was the best way to prevent motion and minimize differences due to PVE. Not controlling the count rate between scans is another limitation because scatter fraction increases slightly with count rate (68). Even if the coincidence prompt rate were kept constant (i.e. by varying the source activity), the event rate in the detector closest to the point source would inevitably vary with the source activity. Considering these limitations, the most accurate way to quantify the effects of scatter and attenuation would be with Monte Carlo simulations.

\section{Future Work}

Further investigations should include scans with activity outside the FOV to simulate the rate of randoms from uptake in normal tissue. Lesions of different sizes should be included and they should be evaluated at multiple locations within the FOV. The errors due to attenuation and scatter should be evaluated at multiple locations within the FOV.

Considering the difficulties in isolating the individual sources of error, the most accurate way to quantify the effects of count rate effects, scatter and attenuation may be to use Monte Carlo simulations. 


\section{Conclusions}

The total quantitative error of the PEM Flex Solo II has been shown to be quite large in the uniform background, lesions and cysts in gelatin breast phantoms of different thicknesses. This system shares many sources of quantitative error with conventional PET scanners, including count rate effects, attenuation and scatter. In addition, the planar LAT reconstruction further degrades the quantitative accuracy in lesions and cysts.

The total error increases negatively with increasing phantom thickness. The error in lesions also increases non-linearly with LBR, probably due to LAT. The effects of LAT on lesion error are important if the ultimate goal of the PEM Flex Solo II is accurate quantitation to facilitate diagnosis of lesions.

The overall error is greatly affected by the count rate, even at clinically relevant background activity concentration and LBRs. The count rate error is different in lesions and background, due to the contribution of signal to the lesions from the background; another effect of LAT. The count rate performance of the PEM Flex scanner will need to be thoroughly characterized and corrections for dead time and randoms will need to be developed in order to improve the quantitative accuracy of this system.

The error due to attenuation by even small breasts is quite large, and it increases with thickness. This error is heavily weighted by oblique LORs with longer path lengths than direct LORs. Attenuation is clearly a substantial source of error for which corrections need to be employed on the PEM Flex system. Monte Carlo investigations may be necessary for developing and evaluating accurate corrections for attenuation to account for small angle scatter, count rate effects, and location within the image volume. The attenuation correction algorithm should be performed on projection data rather than 
image data, due to the dependence of attenuation along LORs and angle of incidence.

Scatter introduced a substantial positive error which appeared to be independent of thickness. Corrections for scatter will be essential for the PEM Flex Solo II to be quantitatively accurate. Monte Carlo investigations may also be necessary to evaluate and develop corrections for scatter, for the same reasons as mentioned above.

Despite the limitations of this study, the hypothesis has been shown to be true for most of the phantom and lesion sizes evaluated. The quantitative error in images of the PEM Flex Solo was greater than $30 \%$ in lesions with a 5:1 LBR or greater in 4 to $12-\mathrm{cm}$ thick breast phantoms, and in lesions with a $2: 1 \mathrm{LBR}$ in 6 to $12-\mathrm{cm}$ thick phantoms. The total quantitative error was influence largely by the effects investigated by each specific aim. Count rate, attenuation and scatter each contributed substantial amounts of error. LAT was also indicated as a major source of error in lesions, but was not specifically investigated as part of this thesis.

While this system has been used to detect early stage breast cancers with high sensitivity and specificity, its diagnostic capabilities will be limited by its low quantitative accuracy. Corrections for count rate, attenuation and scatter are routinely applied in conventional PET imaging and should be adapted to the planar geometry used by the PEM Flex Solo II. LAT, as well as other sources of error (e.g. detector normalization), will continue to be an obstacle to accurate quantitation with this system. 


\section{REFERENCES}

1. 2010. United States Cancer Statistics: 1999-2006 Incidence and Mortality Webbased Report. U.S. Department of Health and Human Services, Centers for Disease Control and Prevention and National Cancer Institute, Atlanta.

2. Altekruse SF, K. C., Krapcho M, Neyman N, Aminou R, Waldron W, Ruhl J, Howlader N, Tatalovich Z, Cho H, Mariotto A, Eisner MP, Lewis DR, Cronin K, Chen HS, Feuer EJ, Stinchcomb DG, Edwards BK (eds). . 2010. SEER Cancer Statistics Review, 1975-2007. National Cancer Institute, Bethesda, MD.

3. Smith, R. A., V. Cokkinides, D. Brooks, D. Saslow, and O. W. Brawley. 2010. Cancer screening in the United States, 2010: a review of current American Cancer Society guidelines and issues in cancer screening. CA: a cancer journal for clinicians 60:99-119.

4. Tabar, L., B. Vitak, H. H. Chen, M. F. Yen, S. W. Duffy, and R. A. Smith. 2001. Beyond randomized controlled trials: organized mammographic screening substantially reduces breast carcinoma mortality. Cancer 91:1724-1731.

5. Group, T. S. O. S. S. E. 2006. Reduction in breast cancer mortality from organized service screening with mammography: 1. Further confirmation with extended data. Cancer Epidemiol Biomarkers Prev 15:45-51.

6. Kolb, T. M., J. Lichy, and J. H. Newhouse. 2002. Comparison of the performance of screening mammography, physical examination, and breast US and evaluation of factors that influence them: an analysis of 27,825 patient evaluations. Radiology 225:165-175. 
7. Berg, W. A., J. D. Blume, J. B. Cormack, E. B. Mendelson, D. Lehrer, M. BohmVelez, E. D. Pisano, R. A. Jong, W. P. Evans, M. J. Morton, M. C. Mahoney, L. H. Larsen, R. G. Barr, D. M. Farria, H. S. Marques, and K. Boparai. 2008. Combined screening with ultrasound and mammography vs mammography alone in women at elevated risk of breast cancer. Jama 299:2151-2163.

8. Benard, F., and E. Turcotte. 2005. Imaging in breast cancer: Single-photon computed tomography and positron-emission tomography. Breast Cancer Res 7:153-162.

9. Salomon, A. 1913. Beiträge zur Pathologie und Klinik der Mammakarzinome. Archiv für Klinische Chirurgie 101:16.

10. Warren, S. L. 1930. A Roentgenologic Study of the Breast. The American journal of roentgenology, radium therapy, and nuclear medicine 24:12.

11. Bassett, L. W., and R. H. Gold. 1988. The evolution of mammography. Ajr 150:493-498.

12. Mandelson, M. T., N. Oestreicher, P. L. Porter, D. White, C. A. Finder, S. H. Taplin, and E. White. 2000. Breast density as a predictor of mammographic detection: comparison of interval- and screen-detected cancers. Journal of the National Cancer Institute 92:1081-1087.

13. Pisano, E. D., C. Gatsonis, E. Hendrick, M. Yaffe, J. K. Baum, S. Acharyya, E. F. Conant, L. L. Fajardo, L. Bassett, C. D'Orsi, R. Jong, and M. Rebner. 2005. Diagnostic performance of digital versus film mammography for breast-cancer screening. The New England journal of medicine 353:1773-1783. 
14. Pisano, E. D., R. E. Hendrick, M. J. Yaffe, J. K. Baum, S. Acharyya, J. B. Cormack, L. A. Hanna, E. F. Conant, L. L. Fajardo, L. W. Bassett, C. J. D'Orsi, R. A. Jong, M. Rebner, A. N. Tosteson, and C. A. Gatsonis. 2008. Diagnostic accuracy of digital versus film mammography: exploratory analysis of selected population subgroups in DMIST. Radiology 246:376-383.

15. Silverstein, M. J., A. Recht, M. D. Lagios, I. J. Bleiweiss, P. W. Blumencranz, T. Gizienski, S. E. Harms, J. Harness, R. J. Jackman, V. S. Klimberg, R. Kuske, G. M. Levine, M. N. Linver, E. A. Rafferty, H. Rugo, K. Schilling, D. Tripathy, F. A. Vicini, P. W. Whitworth, and S. C. Willey. 2009. Special report: Consensus conference III. Image-detected breast cancer: state-of-the-art diagnosis and treatment. Journal of the American College of Surgeons 209:504-520.

16. Sickles, E. A., R. A. Filly, and P. W. Callen. 1984. Benign breast lesions: ultrasound detection and diagnosis. Radiology 151:467-470.

17. Stavros, A. T., D. Thickman, C. L. Rapp, M. A. Dennis, S. H. Parker, and G. A. Sisney. 1995. Solid breast nodules: use of sonography to distinguish between benign and malignant lesions. Radiology 196:123-134.

18. Hong, A. S., E. L. Rosen, M. S. Soo, and J. A. Baker. 2005. BI-RADS for sonography: positive and negative predictive values of sonographic features. Ajr 184:1260-1265.

19. Evans, A. J., S. E. Pinder, D. R. Snead, A. R. Wilson, I. O. Ellis, and C. W. Elston. 1997. The detection of ductal carcinoma in situ at mammographic screening enables the diagnosis of small, grade 3 invasive tumours. British journal of cancer 75:542-544. 
20. Buchberger, W., A. Niehoff, P. Obrist, P. DeKoekkoek-Doll, and M. Dunser. 2000. Clinically and mammographically occult breast lesions: detection and classification with high-resolution sonography. Seminars in ultrasound, CT, and MR 21:325-336.

21. Crystal, P., S. D. Strano, S. Shcharynski, and M. J. Koretz. 2003. Using sonography to screen women with mammographically dense breasts. Ajr 181:177182.

22. Gordon, P. B., and S. L. Goldenberg. 1995. Malignant breast masses detected only by ultrasound. A retrospective review. Cancer 76:626-630.

23. Kaplan, S. S. 2001. Clinical utility of bilateral whole-breast US in the evaluation of women with dense breast tissue. Radiology 221:641-649.

24. Leconte, I., C. Feger, C. Galant, M. Berliere, B. V. Berg, W. D'Hoore, and B. Maldague. 2003. Mammography and subsequent whole-breast sonography of nonpalpable breast cancers: the importance of radiologic breast density. Ajr 180:1675-1679.

25. de Bresser, J., B. de Vos, F. van der Ent, and K. Hulsewe. 2010. Breast MRI in clinically and mammographically occult breast cancer presenting with an axillary metastasis: a systematic review. Eur J Surg Oncol 36:114-119.

26. Eby, P. R., and C. D. Lehman. 2008. Magnetic resonance imaging--guided breast interventions. Top Magn Reson Imaging 19:151-162.

27. Orel, S. G., and M. D. Schnall. 2001. MR Imaging of the Breast for the Detection, Diagnosis, and Staging of Breast Cancer1. 13-30. 
28. Liberman, M., F. Sampalis, D. S. Mulder, and J. S. Sampalis. 2003. Breast cancer diagnosis by scintimammography: a meta-analysis and review of the literature. Breast cancer research and treatment 80:115-126.

29. Bender, H., E. Friedrich, P. O. Zamora, S. Guhlke, and H. J. Biersack. 1997. Effects of induction of multi-drug resistance on accumulation of 99mTc-sestamibi in vitro. Anticancer research 17:1833-1839.

30. Chiu, M. L., J. F. Kronauge, and D. Piwnica-Worms. 1990. Effect of mitochondrial and plasma membrane potentials on accumulation of hexakis (2methoxyisobutylisonitrile) technetium(I) in cultured mouse fibroblasts. J Nucl Med 31:1646-1653.

31. Maublant, J. C., Z. Zhang, M. Rapp, M. Ollier, J. Michelot, and A. Veyre. 1993. In vitro uptake of technetium-99m-teboroxime in carcinoma cell lines and normal cells: comparison with technetium-99m-sestamibi and thallium-201. J Nucl Med 34:1949-1952.

32. Piwnica-Worms, D., M. L. Chiu, M. Budding, J. F. Kronauge, R. A. Kramer, and J. M. Croop. 1993. Functional imaging of multidrug-resistant P-glycoprotein with an organotechnetium complex. Cancer research 53:977-984.

33. Taillefer, R. 1999. The role of 99mTc-sestamibi and other conventional radiopharmaceuticals in breast cancer diagnosis. Seminars in nuclear medicine 29:16-40.

34. Waxman, A. D. 1997. The role of $(99 \mathrm{~m}) \mathrm{Tc}$ methoxyisobutylisonitrile in imaging breast cancer. Seminars in nuclear medicine 27:40-54. 
35. O'Connor, M. K., S. W. Phillips, C. B. Hruska, D. J. Rhodes, and D. A. Collins. 2007. Molecular breast imaging: advantages and limitations of a scintimammographic technique in patients with small breast tumors. The breast journal 13:3-11.

36. Avril, N., M. Menzel, J. Dose, M. Schelling, W. Weber, F. Janicke, W. Nathrath, and M. Schwaiger. 2001. Glucose metabolism of breast cancer assessed by 18FFDG PET: histologic and immunohistochemical tissue analysis. J Nucl Med 42:916.

37. Rose, C., J. Dose, and N. Avril. 2002. Positron emission tomography for the diagnosis of breast cancer. Nuclear medicine communications 23:613-618.

38. Carkaci, S., H. A. Macapinlac, M. Cristofanilli, O. Mawlawi, E. Rohren, A. M. Gonzalez Angulo, S. Dawood, E. Resetkova, H. T. Le-Petross, and W. T. Yang. 2009. Retrospective study of 18F-FDG PET/CT in the diagnosis of inflammatory breast cancer: preliminary data. J Nucl Med 50:231-238.

39. Schwarz-Dose, J., M. Untch, R. Tiling, S. Sassen, S. Mahner, S. Kahlert, N. Harbeck, A. Lebeau, W. Brenner, M. Schwaiger, F. Jaenicke, and N. Avril. 2009. Monitoring primary systemic therapy of large and locally advanced breast cancer by using sequential positron emission tomography imaging with [18F]fluorodeoxyglucose. J Clin Oncol 27:535-541.

40. Schegerin, M., A. N. Tosteson, P. A. Kaufman, K. D. Paulsen, and B. W. Pogue. 2009. Prognostic imaging in neoadjuvant chemotherapy of locally-advanced breast cancer should be cost-effective. Breast cancer research and treatment 114:537-547. 
41. Thompson, C. J., K. Murthy, I. N. Weinberg, and F. Mako. 1994. Feasibility study for positron emission mammography. Medical physics 21:529-538.

42. Abreu, M. C., P. Almeida, F. Balau, N. C. Ferreira, S. Fetal, F. Fraga, M. Martins, N. Matela, R. Moura, C. Ortigao, L. Peralta, P. Rato, R. Ribeiro, P. Rodrigues, A. I. Santos, A. Trindade, and J. Varela. 2005. Clear-PEM: a dedicated PET camera for improved breast cancer detection. Radiation protection dosimetry 116:208210.

43. Doshi, N. K., Y. Shao, R. W. Silverman, and S. R. Cherry. 2000. Design and evaluation of an LSO PET detector for breast cancer imaging. Medical physics 27:1535-1543.

44. Raylman, R. R., S. Majewski, R. Wojcik, A. G. Weisenberger, B. Kross, V. Popov, and H. A. Bishop. 2000. The potential role of positron emission mammography for detection of breast cancer. A phantom study. Medical physics 27:1943-1954.

45. MacDonald, L., J. Edwards, T. Lewellen, D. Haseley, J. Rogers, and P. Kinahan. 2009. Clinical imaging characteristics of the positron emission mammography camera: PEM Flex Solo II. J Nucl Med 50:1666-1675.

46. Karimian, A., C. J. Thompson, S. Sarkar, G. Raisali, R. Pani, H. Davilu, and D. Sardari. 2005. CYBPET: a cylindrical PET system for breast imaging. Nuclear Instruments and Methods in Physics Research Section A: Accelerators, Spectrometers, Detectors and Associated Equipment 545:427-435.

47. Bowen, S. L., Y. Wu, A. J. Chaudhari, L. Fu, N. J. Packard, G. W. Burkett, K. Yang, K. K. Lindfors, D. K. Shelton, R. Hagge, A. D. Borowsky, S. R. Martinez, 
J. Qi, J. M. Boone, S. R. Cherry, and R. D. Badawi. 2009. Initial characterization of a dedicated breast PET/CT scanner during human imaging. J Nucl Med 50:1401-1408.

48. Weidong, L., E. Anashkin, and C. G. Matthews. 2008. Performance Evaluation of a PEM Scanner Using the NEMA NU 4-2008 Small Animal PET Standards. Nuclear Science, IEEE Transactions on 57:94-103.

49. Cherry, S. R., J. A. Sorenson, and M. E. Phelps. 2003. Physics in Nuclear Medicine. Saunders, Philadelphia, PA.

50. Weinberg, I. N., D. Beylin, V. Zavarzin, S. Yarnall, P. Y. Stepanov, E. Anashkin, D. Narayanan, S. Dolinsky, K. Lauckner, and L. P. Adler. 2005. Positron emission mammography: high-resolution biochemical breast imaging. Technology in cancer research \& treatment 4:55-60.

51. Tafra, L., Z. Cheng, J. Uddo, M. B. Lobrano, W. Stein, W. A. Berg, E. Levine, I. N. Weinberg, D. Narayanan, E. Ross, D. Beylin, S. Yarnall, R. Keen, K. Sawyer, J. Van Geffen, R. L. Freimanis, E. Staab, L. P. Adler, J. Lovelace, P. Shen, J. Stewart, and S. Dolinsky. 2005. Pilot clinical trial of 18F-fluorodeoxyglucose positron-emission mammography in the surgical management of breast cancer. American journal of surgery 190:628-632.

52. Berg, W. A., I. N. Weinberg, D. Narayanan, M. E. Lobrano, E. Ross, L. Amodei, L. Tafra, L. P. Adler, J. Uddo, W. Stein, 3rd, and E. A. Levine. 2006. Highresolution fluorodeoxyglucose positron emission tomography with compression ("positron emission mammography") is highly accurate in depicting primary breast cancer. The breast journal 12:309-323. 
53. Raylman, R. R., S. Majewski, A. G. Weisenberger, V. Popov, R. Wojcik, B. Kross, J. S. Schreiman, and H. A. Bishop. 2001. Positron emission mammography-guided breast biopsy. J Nucl Med 42:960-966.

54. Macdonald, L., J. Edwards, T. Lewellen, J. Rogers, and P. Kinahan. 2008. Clinical Imaging Characteristics of the Positron Emission Mammography PEM Flex Solo II. IEEE Nuclear Science Symposium conference record 11:4494-4501.

55. Wahl, R. L., H. Jacene, Y. Kasamon, and M. A. Lodge. 2009. From RECIST to PERCIST: Evolving Considerations for PET response criteria in solid tumors. J Nucl Med 50 Suppl 1:122S-150S.

56. Vranjesevic, D., C. Schiepers, D. H. Silverman, A. Quon, J. Villalpando, M. Dahlbom, M. E. Phelps, and J. Czernin. 2003. Relationship between 18F-FDG uptake and breast density in women with normal breast tissue. J Nucl Med 44:1238-1242.

57. Shkumat, N., E. Rohren, W. T. Yang, B. E. Adrada, E. Arribas, S. Carkaci, H. H. Chuang, L. Santiago, and O. Mawlawi. Investigating the limit of detectability of a positron emission mammography device; a phantom study. European Journal of Nuclear Medicine and Molecular Imaging Under review.

58. Berger, M. J., Hubbell, J.H., Seltzer, S.M., Chang, J., Coursey, J.S., Sukumar, R., and Zucker, D.S. 2005. XCOM: Photon Cross Section Database (version 1.3). National Institute of Standards and Technology, Gaithersburg, MD.

59. Watson, C. C., M. E. Casey, B. Bendriem, J. P. Carney, D. W. Townsend, S. Eberl, S. Meikle, and F. P. Difilippo. 2005. Optimizing injected dose in clinical 
PET by accurately modeling the counting-rate response functions specific to individual patient scans. J Nucl Med 46:1825-1834.

60. Ogden, C. L., C. D. Fryar, M. D. Carroll, and K. M. Flegal. 2004. Mean body weight, height, and body mass index, United States 1960-2002. Advance data:117.

61. Zasadny, K. R., and R. L. Wahl. 1993. Standardized uptake values of normal tissues at PET with 2-[fluorine-18]-fluoro-2-deoxy-D-glucose: variations with body weight and a method for correction. Radiology 189:847-850.

62. Bailey, D. M., Townsend, D. W. Valk, P. E., Maisey, M. N. (Eds). 2005. Positron Emission Tomography. Springer-Verlag, London.

63. Bailey, D. L., and S. R. Meikle. 1994. A convolution-subtraction scatter correction method for 3D PET. Physics in medicine and biology 39:411-424.

64. Murthy, K., M. Aznar, C. J. Thompson, A. Loutfi, R. Lisbona, and J. H. Gagnon. 2000. Results of preliminary clinical trials of the positron emission mammography system PEM-I: a dedicated breast imaging system producing glucose metabolic images using FDG. J Nucl Med 41:1851-1858.

65. Compton, A. H. 1923. The Quantum Integral and Diffraction by a Crystal. Proceedings of the National Academy of Sciences of the United States of America $9: 359-362$.

66. Raylman, R. R., S. Majewski, R. Wojcik, A. G. Weisenberger, B. Kross, and V. Popov. 2001. Corrections for the Effects of Accidental Coincidences, Compton Scatter, and Object Size in Positron Emission Mammography (PEM) Imaging. IEEE Transaction on Nuclear Science 48:11. 
67. Murthy, K., D. Jolly, M. Aznar, C. J. Thompson, P. Sciascia, A. Loutfi, R. Lisbona, and J. H. Gagnon. 1999. Quantification in Positron Emission Mammography (PEM) with Planar Detectors: Contrast Resolution Measurements using a Custom Breast Phantom and Novel Sperical Hot-Spots. IEEE Transaction on Nuclear Science 46:5.

68. Erdi, Y. E., S. A. Nehmeh, T. Mulnix, J. L. Humm, and C. C. Watson. 2004. PET performance measurements for an LSO-based combined PET/CT scanner using the National Electrical Manufacturers Association NU 2-2001 standard. J Nucl Med 45:813-821. 


\section{VITA}

Adam Christopher Springer was born in Wichita Falls, Texas on January 23, 1980 to Bob and Therese Springer. He earned in his Bachelor of Science degree in Physics at the University of Southern Mississippi in Hattiesburg, Mississippi in May 2004. He worked as a Physics Technologist before matriculating in The University of Texas Health Science Center at Houston, Graduate School of Biomedical Sciences in August of 2004.

Permanent Address:

6570 Cross Creek Trail

Brecksville, Ohio 44141 